\title{
Near-Field Mapping of Photonic Eigenmodes in Patterned Silicon Nanocavities by Electron Energy-Loss Spectroscopy
}

\author{
Duncan T. L. Alexander,* Valentin Flauraud, and Frank Demming-Janssen
}

Cite This: https://doi.org/10.1021/acsnano.1c06065

Read Online

ACCESS 1

山ll Metrics \& More

回国 Article Recommendations

SI Supporting Information

ABSTRACT: Recently, there has been significant interest in using dielectric nanocavities for the controlled scattering of light, owing to the diverse electromagnetic modes that they support. For plasmonic systems, electron energy-loss spectroscopy (EELS) is now an established method enabling structureoptical property analysis at the scale of the nanostructure. Here, we instead test its potential for the near-field mapping of photonic eigenmodes supported in planar dielectric nanocavities, which are lithographically patterned from amorphous silicon according to standard photonic principles. By correlating results with finite element simulations, we demonstrate how many of the EELS excitations can be directly corresponded to
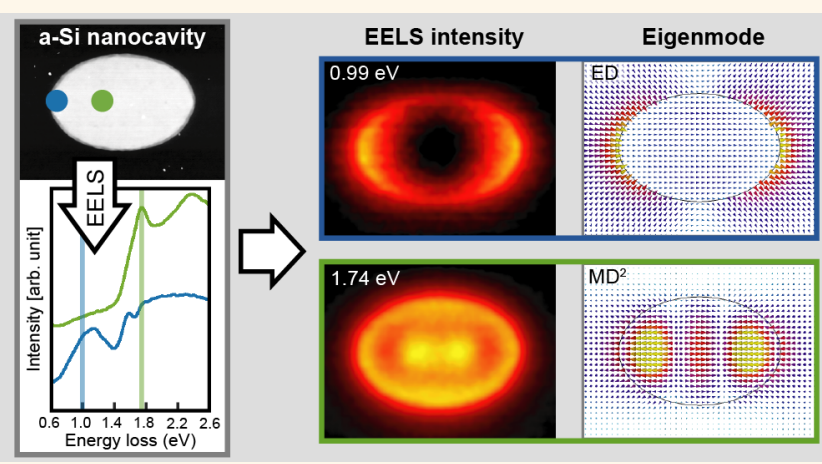
various optical eigenmodes of interest for photonic engineering.

The EELS maps present a high spatial definition, displaying intensity features that correlate precisely to the impact parameters giving the highest probability of modal excitation. Further, eigenmode characteristics translate into their EELS signatures, such as the spatially and energetically extended signal of the low $Q$-factor electric dipole and nodal intensity patterns emerging from excitation of toroidal and second-order magnetic modes within the nanocavity volumes. Overall, the spatial-spectral nature of the data, combined with our experimental-simulation toolbox, enables interpretation of subtle changes in the EELS response across a range of nanocavity dimensions and forms, with certain simulated resonances matching the excitation energies within $\mathbf{\pm 0 . 0 1} \mathrm{eV}$. By connecting results to far-field simulations, perspectives are offered for tailoring the nanophotonic resonances via manipulating nanocavity size and shape.

KEYWORDS: STEM-EELS, dielectric, eigenmode, nanocavity, resonator, CST Studio Suite

ince the early reports showing two-dimensional (2D) mapping of localized surface plasmon resonances (LSPRs) using scanning transmission electron microscopy (STEM)-based electron energy-loss spectroscopy (EELS), ${ }^{1,2}$ this technique has become an established tool for the near-field imaging of plasmonic optical excitations. Applications of this approach (or its energy filtered transmission electron microscopy equivalent) include: mapping and interpretation of resonances in different geometric planar shapes; ${ }^{3-6}$ the measurement of coupled modes between metallic nanoparticles ${ }^{7-11}$ or nanorods; ${ }^{12-14}$ the illustration of Babinet's principle and the study of fractal effects; ${ }^{15-18}$ and extension to the three-dimensional reconstruction of coupled plasmonic fields and photonic environments. ${ }^{19-21}$ Moreover, the technique has been applied to provide evidence for selfhybridization within non-Hermitian LSPR systems ${ }^{22}$ and enabled the nanoscale $2 \mathrm{D}$ visualization of plasmon-exciton polaritons between silver nanotriangles and $\mathrm{WS}_{2}$ flakes. ${ }^{23}$
Allied to these experimental works, progress in theoretical understanding and simulations has been vital for interpretation of results. ${ }^{24-30}$ Even if the energy resolution offered by STEMEELS is lower than that of light-based techniques, the association of spectral signatures to their $2 \mathrm{D}$ projected intensity maps gives the technique a high sensitivity to the effects of subtle structural modifications, as for example shown in studies of heterodimers and dolmen structures. ${ }^{10,14}$ While it is considered as a near-field mapping technique, it should be noted that the maps of LSPRs do not necessarily bear a one-to-

Received: July 17, 2021

Accepted: September 23, 2021 
one correspondence with the local electric field of the excited mode. ${ }^{25}$ Rather, the EELS signal is maximal at probe impact parameters giving the highest probability of exciting a mode which, in cases such as the bonding mode of a dimer, can be quite different to where the mode's projected electric field is strongest.

One of the motivations for the study of LSPRs is their use in optical nanoantennas, where electromagnetic (EM) fields are manipulated on the subwavelength scale by coupling the propagating electric field of light with the local electric field of the nanostructure. ${ }^{31,32}$ However, strong absorption losses of metals at infrared-visible optical frequencies limit the performance of LSPR-based nanoantennas in applications where light scattering is the goal. This can be solved by turning to an alternative strategy in which the Mie-type resonances of high-permittivity dielectric nanoparticles or nanostructures are interfaced with the propagating and local EM fields. ${ }^{33}$ Because the Mie-type resonances are driven by displacement currents originating from the polarization of atoms (or molecules) within the dielectric volume, rather than the LSPR case of actual currents of charge on the nanostructure surface, they offer reduced losses. ${ }^{33,34}$ The dielectric systems also introduce an extra degree of freedom for manipulating light, since they can support both electric and magnetic resonant eigenmodes. For instance, unlike the purely electric modes of equivalently shaped plasmonic nanoparticles, dielectric spheres or discs exhibit magnetic dipole (MD) resonances deriving from the excitation of circular displacement currents within a nanoparticle, which induce a MD orthogonal to the current loop. ${ }^{35,36}$ As example applications, the simultaneous excitation and mutual interference of electric dipole (ED) and MD resonances enables directional visible light scattering with zero backscattering from single nanoparticles, via the Kerker effect, while the interference of the ED with a toroidal dipole can give a sharp dip in forward scattering known as the anapole. ${ }^{39-42}$ By using silicon as the dielectric, a further benefit over plasmonics for large-scale implementation comes from its straightforward integration into standard microfabrication processes. $^{43}$

With EELS mapping now an established tool enabling the structure-optical property analysis of plasmonic systems at the scale of the nanostructure, this work was motivated by the question: can it do the same for dielectric nanocavities? Little has been published concerning use of EELS for measuring the optical excitations in nanostructured dielectric systems. After Garcia de Abajo et al. showed that a fast electron can be used as a probe of photonic bands in photonic crystal structures, via the Vavilov-Cherenkov effect, ${ }^{44}$ this approach was used to map the local density of states in the void regions of silicon photonic structures. ${ }^{45}$ Le Thomas et al. demonstrated that this concept can be further adapted to image the cavity optical modes in bulk material, in the study of a three missing hole defect in a hexagonal photonic lattice. ${ }^{46}$ Such measurements are, however, quite different to probing Mie-type resonances or cavity modes in individual dielectric nanoparticles. Until now, the near-field mapping of such localized optical modes in silicon nanostructures has been demonstrated using apertureless near-field optical microscopy, ${ }^{47}$ and with cathodoluminescence in lower beam-energy scanning electron microscopy $^{36,48,49}$ and higher beam energy STEM. ${ }^{50}$ In comparison, here we explore the potential for using STEM-EELS to map such resonances in patterned structures of varied geometry, which are designed according to standard photonic processes.
Our analysis brings insights both into the nature of the optical structures and for the field of EELS.

The samples in our study are planar amorphous-silicon (a$\mathrm{Si}$ ) nanocavities of different geometries, patterned by top-down electron beam lithographic processing. The particle sizes are tuned to give resonances at frequencies from the near-infrared through visible regime with, for instance, the discs being similar to those with potential for nanoscale color printing via tuned light scattering. ${ }^{43}$ Considering that the excitations are probed in samples with a total of $135 \mathrm{~nm}$ thickness, we apply a high electron beam energy of $300 \mathrm{keV}$ in order to improve the signal-to-noise ratio in the EELS peaks, by reducing scattering to the bulk plasmon at higher-energy losses. This differs to recent work by Liu et al. on EELS of cavity modes in colloidal oxide nanocubes, where low incident beam energies of 40 or $60 \mathrm{keV}$ effectively restricted analysis to an "aloof" geometry, in which the electron probe is positioned some nanometers outside of the nanostructure. ${ }^{51}$ Such an approach precludes the potential for $2 \mathrm{D}$ mapping of different excitations within the nanocavity body, as we demonstrate here.

Dielectric nanocavities can support a wide range of EM resonances. Further, similarly to the excitation of "dark" modes in plasmonic structures, $3,5,7-10,18,52$ excitation via the local field of the electron probe means that STEM-EELS gives access to many modes which cannot be straightforwardly excited with planewave EM illumination. In order to interpret the STEMEELS data, different excitations are identified from their spatial and spectral signatures and corresponded to specific eigenmodes, as simulated using CST Studio Suite. ${ }^{53}$ With this combined spectroscopy and simulation strategy, we explore the effects of both nanocavity size and shape on the excitations. We also discuss how the EELS response of the dielectric nanostructures contrasts with that for plasmonic systems.

\section{RESULTS AND DISCUSSION}

$100 \mathrm{~nm}$ thick a-Si nanocavities of varying shapes (discs, ellipses, rectangles, square, and triangle), and supported on 35 $\mathrm{nm}$ thick electron transparent $\mathrm{Si}_{3} \mathrm{~N}_{4}$ membranes, were mapped in $2 \mathrm{D}$ using monochromated STEM-EELS. (See Methods section for details.) In the frequency range of interest, the dielectric permittivity of the a-Si is $\sim 14-26$, compared with $\sim 4$ for the $\mathrm{Si}_{3} \mathrm{~N}_{4}$. As a first example of data from the samples, Figure 1 shows EEL spectra integrated from four different impact parameters across a $450 \mathrm{~nm}$ diameter a-Si disc. Moving from the edge toward the center, distinct peaks are observed in the spectra, as different resonances are excited by the electron probe. Some key features are indicated by the dashed lines. In the dark blue spectrum from the edge region, two strong peaks at $\sim 1.0$ and $1.37 \mathrm{eV}$ are marked. Next, a $1.87 \mathrm{eV}$ and a $1.69 \mathrm{eV}$ peak are, respectively, observed in the light blue and green spectra taken from distances of $\sim 120$ and $\sim 70 \mathrm{~nm}$ from the disc center. Finally, a strong peak at $\sim 1.56 \mathrm{eV}$ is discriminated in the red spectrum from the disc center. At energy losses above the bandgap of the a-Si $(\sim 1.5 \mathrm{eV})$, the EELS peaks sit on a "shelf" of intensity from other loss processes, making their signature weaker. This aspect applies to all of the EELS data presented here. Particularly problematic is if the peak sits on the rise of this shelf, when it appears as a less defined "hump" of intensity on the spectrum. Excitations are therefore identified by a combination of manual inspection of the data, semiautomated Gaussian peak fitting, and correlation to "hotspots" of intensity in the EELS maps. With this, depending 


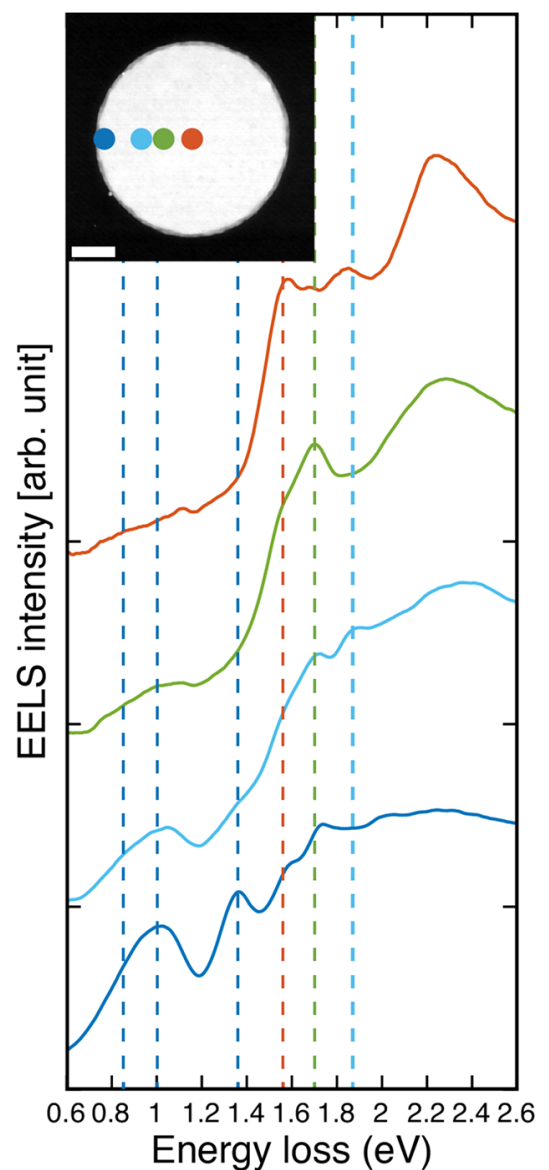

Figure 1. EEL spectra for a $450 \mathrm{~nm}$ a-Si disc. The spectra are integrated from rings at different radii, as indicated using colorcoded spots on the high angle annular dark-field (HAADF) STEM image at the top left, and are displaced on the intensity axis for clarity. Each spectrum is normalized by the intensity of its zeroloss peak (ZLP) and background subtracted for the ZLP and $\mathrm{Si}_{3} \mathrm{~N}_{4}$ membrane. Color-coded vertical dashed lines indicate peaks or features of interest in the spectra, as used to construct the heatintensity maps presented in Figures 2 and 5 . Scale bar: $100 \mathrm{~nm}$.

on the excitation, we estimate a precision of $\pm 0.01-0.02 \mathrm{eV}$ in the EELS peak energy loss values.

Internally Excited Eigenmodes: Disc. In order to interpret the spectral features in Figure 1, we need an analytical approach that gives solutions consistent with cavity modes excited by impacting (as opposed to aloof) electron trajectories. To solve this need, we apply two finite elementbased methods: first, an eigenmode approach to identify likely excited modes; second, as shown in the Supporting Information (SI), frequency domain simulations with localized dipoles to confirm key excitation behavior.

In the eigenmode approach, possible eigenmodes for a nanocavity are simulated using the Eigenmode Solver of CST Studio Suite, as for instance shown for the $450 \mathrm{~nm}$ disc in Figure S2-1 of the SI. ${ }^{53}$ From these, specific eigenmodes are matched to the resonances excited by the electron probe, by considering both the spatial and spectral signatures of the STEM-EELS data. Implicit to this approach is a classical (as opposed to quantum) viewpoint analogous to that commonly used in the interpretation of plasmonic EELS data. ${ }^{24,54,55}$ Namely, that the evanescent electric field produced by the transmitting electron polarizes the nanostructure in the vicinity of its trajectory. The polarization induces an EM resonance, whose electric field $E^{\text {ind }}$ in turn produces a force that acts back on the transmitting electron, giving a peak in the EEL spectrum at an energy loss corresponding to the excited frequency. Compared with the plasmonic case, where the resonance is that of the surface charge, here the excited resonance is instead that of charge displaced within the volume of the nanocavity. In the analysis, inspiration is further taken from the EELS study of bonding and antibonding modes in coupled plasmonic systems, in particular the need for the field of the excited mode to be compatible with the symmetry of the evanescent field of the electron probe that stimulates the excitation. $^{24,25,28,56}$ For instance, the fundamental eigenmode of the $450 \mathrm{~nm}$ disc is an out-of-plane $\mathrm{MD}$, also known as a $\mathrm{TE}_{01 \delta}$ eigenmode. ${ }^{57}$ This has an electric field that rotates around the disc axis, which is fundamentally incompatible with excitation by an electron probe parallel to the axis and so will not be visible in the STEM-EELS data.

To see instead which modes are excited, we first consider the $\sim 1.56 \mathrm{eV}$ peak, which corresponds to a strong, central hotspot, as shown in its EELS intensity map in Figure 2. The electric field of a transmitted electron impacting at the disc center will "push away" or displace the internal charge of the nanocavity in a radially symmetric way. As it enters the upper region of the nanocavity body, or as it leaves the lower part, the resulting excitation geometry is compatible with the electric field distribution of the eigenmode shown below the EELS map. With its poloidal electric field surrounding a toroidal magnetic field-as induced by vertically circulating/radially symmetric displacement current loops-the eigenmode is an out-of-plane toroidal (electric) dipole $\left(\mathrm{TD}_{\mathrm{op}}\right)$, also variously known as an axial $\mathrm{ED}, \mathrm{ED}_{\text {vert }}$ and a $\mathrm{TM}_{01 \delta}$ mode. $^{34,40,42,57,58}$ Its $1.50 \mathrm{eV}$ simulated resonance energy matches the $\sim 1.56 \mathrm{eV}$ EELS peak energy relatively closely, just with a small blue shift of the latter relative to the former. Like for plasmonics, its EELS intensity is maximal at the impact parameter giving the highest probability of exciting the eigenmode, rather than being a direct representation the projected electric field. In order to confirm this modal assignment, we adapt a frequency domain approach previously applied to simulate LSPR EEL spectra, but embedding a vertical exciting dipole within the center of the nanocavity in order to give an excitation geometry approximating that of a centrally placed electron probe. ${ }^{59}$ The resulting plot of electric field response vs energy (see Figure S3-3 in the SI) indeed shows a strong peak at the resonance energy of the $\mathrm{TD}_{\text {op }}$ eigenmode, similarly to the EEL spectrum.

We now consider the $1.70 \mathrm{eV}$ EELS peak observed $\sim 70 \mathrm{~nm}$ from the disc center, as seen on the green curve of Figure 1. The corresponding EELS map in Figure 2 shows a strong ring of intensity at this radius, while there is minimal excitation in the disc center. Comparison to the Eigenmode Solver solutions finds that this excitation corresponds unambiguously to a resonance with three counter-rotating, vertical displacement current loops, as shown below the EELS map. These current loops have straight and aligned axes, giving the characteristics and net magnetic moment of a second-order magnetic dipole $\left(\mathrm{MD}^{2}\right)$, as proposed by Yang et al. and visualized in $\mathrm{Si}$ nanospheres using STEM-CL by Matsukata et al. ${ }^{5060}$ The magnetic analogue of a TD, this mode is degenerate in nature. There is a high probability of exciting this mode when the electron probe impinges at the radius corresponding to the counter rotation of the inner and outer current loops, as shown with a gray dot on the $\mathrm{MD}^{2}$ plan-view field map in Figure 2. By 

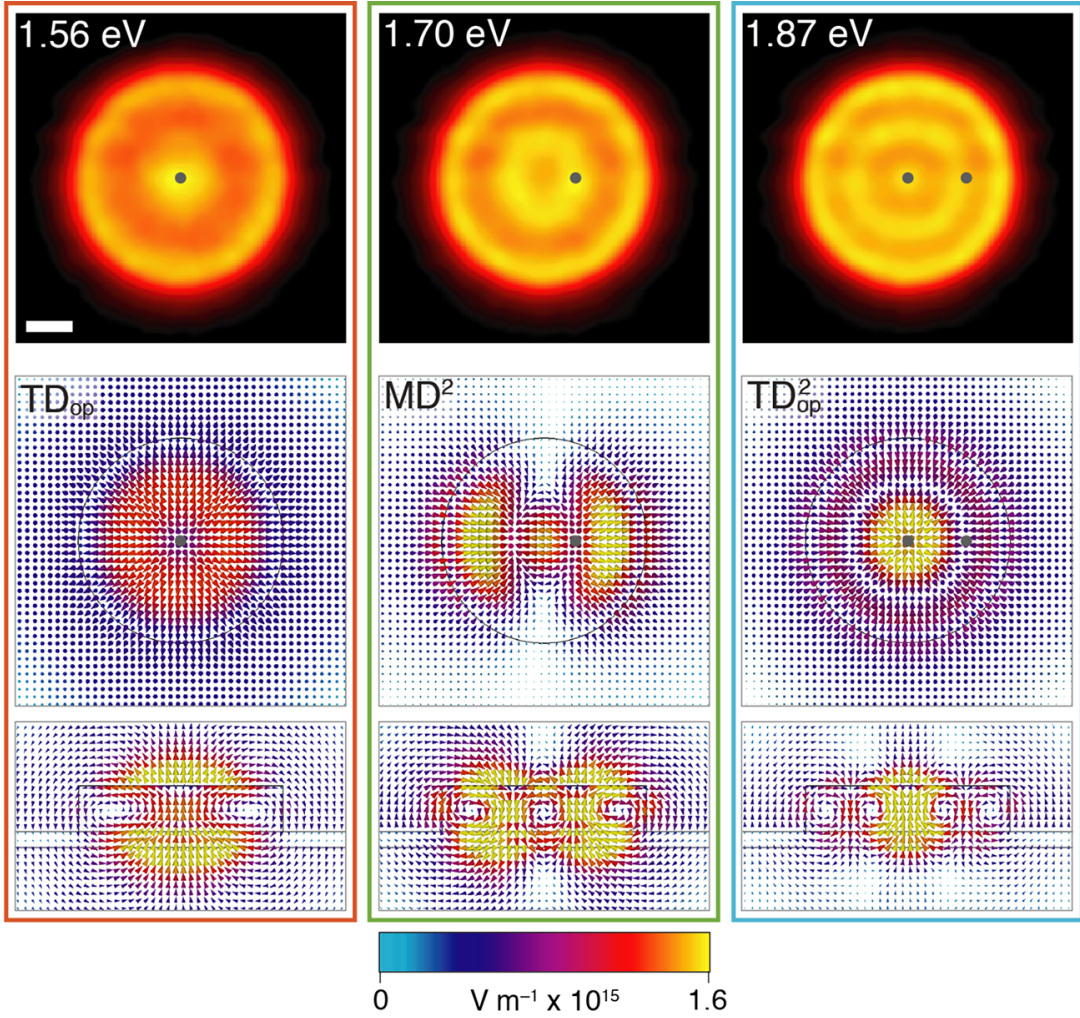

Figure 2. Internally excited eigenmodes for a $450 \mathrm{~nm}$ a-Si disc. The top row shows heat intensity EELS maps integrated across $0.06 \mathrm{eV}$ wide energy windows centered on the indicated energy losses (as for following figures). Each map is corresponded to the excitation of an eigenmode having the electric field distribution shown below it: an out-of-plane toroidal dipole $\left(\mathrm{TD}_{\mathrm{op}}\right)$; a second-order magnetic dipole $\left(\mathrm{MD}^{2}\right)$; and a second-order toroidal dipole $\left(\mathrm{TD}_{\mathrm{op}}^{2}\right)$. The electric field distributions are shown at cut planes corresponding to a height of 85 $\mathrm{nm}$ within the $100 \mathrm{~nm}$ thick discs for the upper plan-view row, and along a horizontal chord going through the disc center for the lower cross-section row. On the plan-view field maps, gray dots indicate the loci expected to give the strongest modal excitation. These are transcribed onto the corresponding EELS maps in order to help relate the two. The outlined boxes are color-coded to the relevant EEL spectra in Figure 1, while the color bar gives the scale for the field maps. Scale bar: $100 \mathrm{~nm}$.

transcribing this locus onto the EELS map, we observe its nanometric alignment with the EELS ring of intensity, supporting the modal assignment. Extra confirmation is provided both by the simulated $\mathrm{MD}^{2}$ resonance energy of $1.69 \mathrm{eV}$ being within $0.01 \mathrm{eV}$ of the EELS peak energy, and by appropriate frequency domain simulations (Figure S3-3 in the SI).

The final internally excited mode that we consider for the $450 \mathrm{~nm}$ disc gives the $1.87 \mathrm{eV}$ peak on the light blue curve in Figure 1. In the corresponding EELS intensity map in Figure 2, it shows both a ring of intensity at the radius of $\sim 120 \mathrm{~nm}$ and a small, central hotspot, and derives from the excitation of the eigenmode shown below. This nondegenerate resonance consists of two in-plane, counter-rotating electric field toroids, with one nested inside the other. Given the nature of the electric far field, we consider this to be a second-order out-ofplane toroidal dipole $\left(\mathrm{TD}_{\mathrm{op}}^{2}\right)$. This eigenmode can be strongly excited both by placing the electron probe at the disc center and at the radius at which the counter-rotating toroids meet, in agreement with the $1.87 \mathrm{eV}$ EELS map. Like the $\mathrm{MD}^{2}$ eigenmode, there is also a close match between the $1.86 \mathrm{eV}$ simulated resonance energy of this eigenmode and the EELS peak energy. A slight misalignment between the locus of expected maximum excitation, as shown with a gray dot in Figure 2, and the ring of intensity in the EELS map could derive from simultaneous excitation of a higher-order $1.85 \mathrm{eV}$ EM mode shown in panel (vii) of SI Figure S2-1 part (b), whose optimum excitation locus would be at a slightly smaller radius; a possibility ideally investigated using a modal decomposition. $^{61}$

Internally Excited Eigenmodes: Ellipse, Rectangle, Triangle, and Square. The $\mathrm{TD}_{\mathrm{op}}$ and $\mathrm{MD}^{2}$ eigenmodes are both strongly visible in the STEM-EELS data from other nanocavity shapes, as illustrated by the intensity maps in Figure 3 for a $450 \times 300 \mathrm{~nm}$ ellipse and rectangle, and $300 \mathrm{~nm}$ side triangle and square. (See SI for full STEM-EELS data.) Figure 3a presents EELS intensity maps with the central, radially symmetric hotspots characteristic of $\mathrm{TD}_{\mathrm{op}}$ excitation. In agreement, the Eigenmode Solver identifies the $\mathrm{TD}_{\mathrm{op}}$ eigenmode in the rectangle, triangle and square. Like for the $450 \mathrm{~nm}$ disc, all show a consistent blue shift of $\sim 0.06-0.08 \mathrm{eV}$ of the EELS peak energy relative to the simulated eigenmode resonance energy. The only exception is the ellipse, for which the $\mathrm{TD}_{\text {op }}$ eigenmode solution is effectively degenerated into two different modes, but whose superposed excitation could lead to the EELS result (see SI Figure S2-4). Lending support to the singular nature of the $\mathrm{TD}_{\mathrm{op}}$, we note that the $\mathrm{TD}_{\mathrm{op}}$ resonance energies from the different shapes correlate closely with those for discs of equivalent circle diameters.

Unlike for the $\mathrm{TD}_{\mathrm{op}}$, nanocavity shape has a strong effect on the degenerate $\mathrm{MD}^{2}$ eigenmode. Since, in the discs, the $\mathrm{MD}^{2}$ mode is equally excited along any radius, it gives a radially symmetric ring of intensity in the EELS map. By going to other forms, the mode becomes constrained to specific axes, leading 

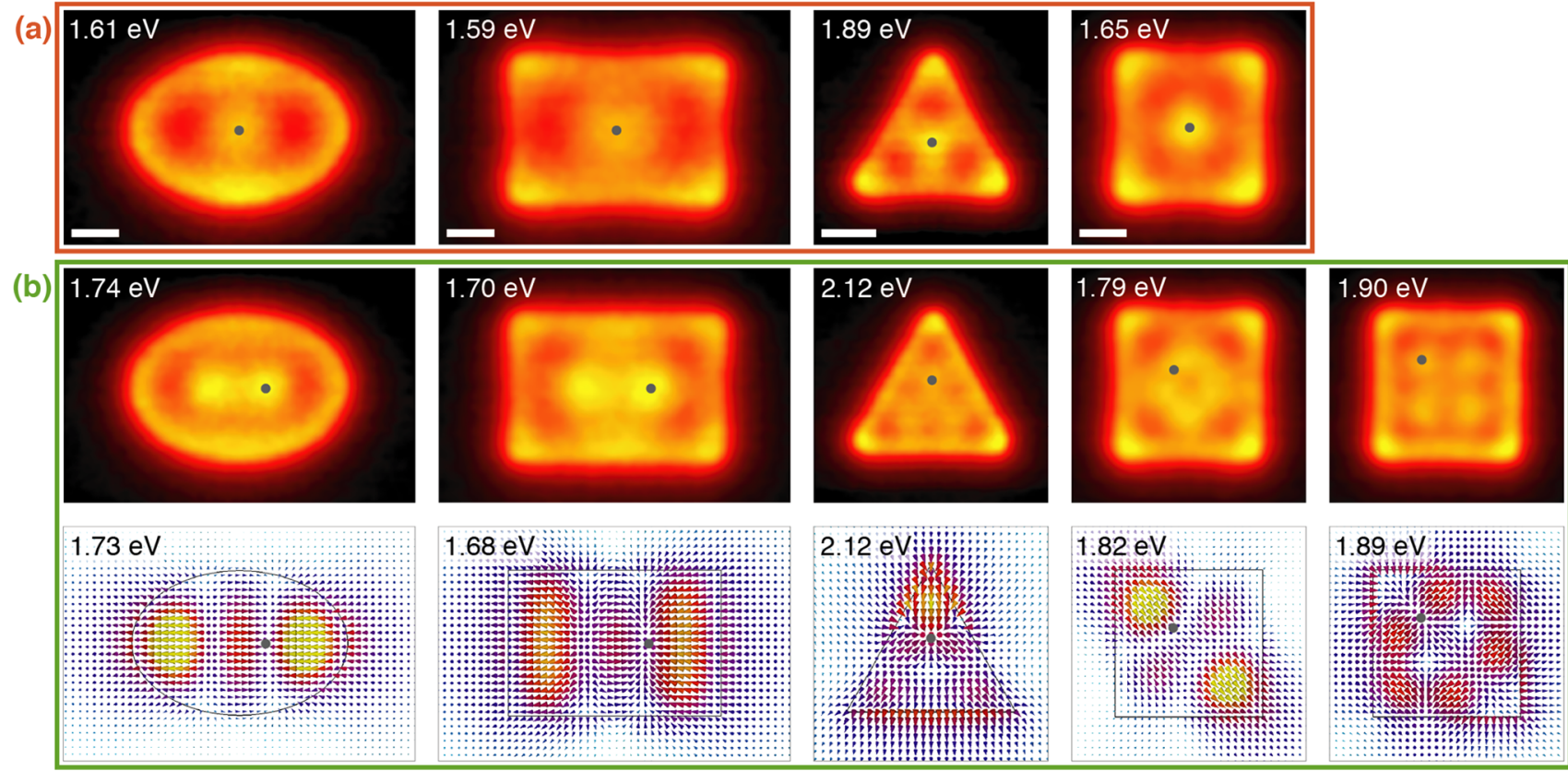

Figure 3. EELS maps and simulated electric field distributions for eigenmodes which are internally excited in a $450 \times 300 \mathrm{~nm}$ ellipse and rectangle, and $300 \mathrm{~nm}$ side triangle and square. Row (a) show the EELS maps corresponding to the excitation of the $T D_{o p}$, as evident from the radially symmetric central hotspots. The upper row in part (b) presents EELS maps for excitation of $\mathrm{MD}^{2}$ eigenmodes. The patterns of intensity hotspots are related to the corresponding $\mathrm{MD}^{2}$ resonance electric field distributions shown in plan-view in the bottom row. The exception is the $1.90 \mathrm{eV}$ map/1.89 eV field distribution pair for the square, which are from a similar but higher-order excitation. Note that the simulated eigenmodes shown respectively account for the formation of the upper hotspot in the $2.12 \mathrm{eV}$ EELS map for the triangle, and the hotspots lying on the top-left to bottom-right diagonals for the 1.79 and $1.90 \mathrm{eV}$ EELS maps for the square. Complementary eigenmodes from their degenerate pairs are responsible for the remaining hotspots (see SI Figures S2-2 and S2-3). The STEM-EELS Data section of the SI presents relevant EEL spectra, color-coded to the outlined boxes in this figure. The electric field distributions are shown at cut planes of $85 \mathrm{~nm}$ height in the nanocavities, as for the plan-view field maps in following figures. The gray dots on the plan-view maps show the examples of the expected loci for the maximum excitation of the corresponding modes, as assessed from their 3D field distributions. These loci are transcribed onto the EELS maps, correcting for any small spatial shifts between the simulated and mapped shapes. The reader is referred to Figures 7 and 8 for respective shape-related color bar scales for the electric field maps. Scale bars: $100 \mathrm{~nm}$.

to patterns of hotspots in the EELS intensity maps, see Figure 3 b. First, considering the ellipse, the deviation from an aspect ratio of one generates an $\mathrm{MD}^{2}$ eigenmode where the three current loops are constrained to lie on the long axis (henceforth termed longitudinal axis) of the nanocavity, as shown in the $1.73 \mathrm{eV}$ electric field distribution in Figure $3 \mathrm{~b}$, with a transverse net magnetic pole. This mode has the highest probability of being excited by the electron probe impacting at the two loci on the longitudinal axis where the counter-rotating electric field loops meet, which explains the two hotspots in the corresponding $1.74 \mathrm{eV}$ EELS map. As an example, the rightside locus is marked with a gray dot. Transcribing this dot onto the $1.74 \mathrm{eV}$ EELS map shows a nanometric coincidence with the right-side EELS hotspot. The $450 \times 300 \mathrm{~nm}$ rectangle shows an equivalent $\mathrm{MD}^{2}$ behavior, as seen in the $1.70 \mathrm{eV}$ EELS map with $1.68 \mathrm{eV}$ simulated eigenmode in Figure $3 \mathrm{~b}$, demonstrating that this nature derives primarily from the nanocavity aspect ratio, not shape. Similar to the $450 \mathrm{~nm}$ disc, as well as the spatial alignment of excitation loci with the EELS hotspots, both the rectangle and ellipse show close agreement between simulated eigenmode resonance and EELS peak energies (see SI Figures S1-4 and S1-5 for the EELS curves). For the longer $600 \times 300 \mathrm{~nm}$ ellipse and rectangle, it appears that the EELS excitation energies of the $\mathrm{MD}^{2}$ and $\mathrm{TD}_{\mathrm{op}}$ eigenmodes converge, such that it is hard to separate them in the EELS data (see Figures S1-6 and S1-7 in the SI.)
With aspect ratios of one, the triangle and square $\mathrm{MD}^{2}$ eigenmodes instead show degenerate pairs which are of equal energy. However, they are still constrained along certain axes. In the triangle, this results in three intensity hotspots in its 2.12 $\mathrm{eV}$ EELS map in Figure 3b, which derive from three strong excitation loci. The latter are split between the pair of eigenmodes, of which the one illustrated below the EELS map accounts for the upper locus, as shown with a gray dot. Interpretation of the $\mathrm{MD}^{2}$ mode in the square is more subtle. The $1.90 \mathrm{eV}$ EELS intensity map in Figure $3 \mathrm{~b}$ in turn shows four hotspots of intensity. However, the $\mathrm{MD}^{2}$-type mode identified by the Eigenmode Solver has a lower energy of 1.82 $\mathrm{eV}$. In the STEM-EELS data, this actually corresponds to an excitation observed in the $1.79 \mathrm{eV}$ map of Figure 3, where the excitation points are so close to the center that they are not well-resolved into four lobes of intensity, but instead show as a more uniform ring of intensity (which is nevertheless wellaligned with the excitation locus indicated with the gray dot). The four-lobed EELS excitation instead corresponds to a higher-order eigenmode. As shown in the $1.89 \mathrm{eV}$ electric field distribution map in Figure 3b, on the diagonal this similarly has three vertical current loops but with excitation loci further from the square center than for the $\mathrm{MD}^{2}$ mode. Elsewhere in the square, this mode has a more complex, higher-order nature than the lower energy $\mathrm{MD}^{2}$.

Edge Excited Eigenmodes: Disc. The above analysis finds that placing the electron probe within the volume of the 

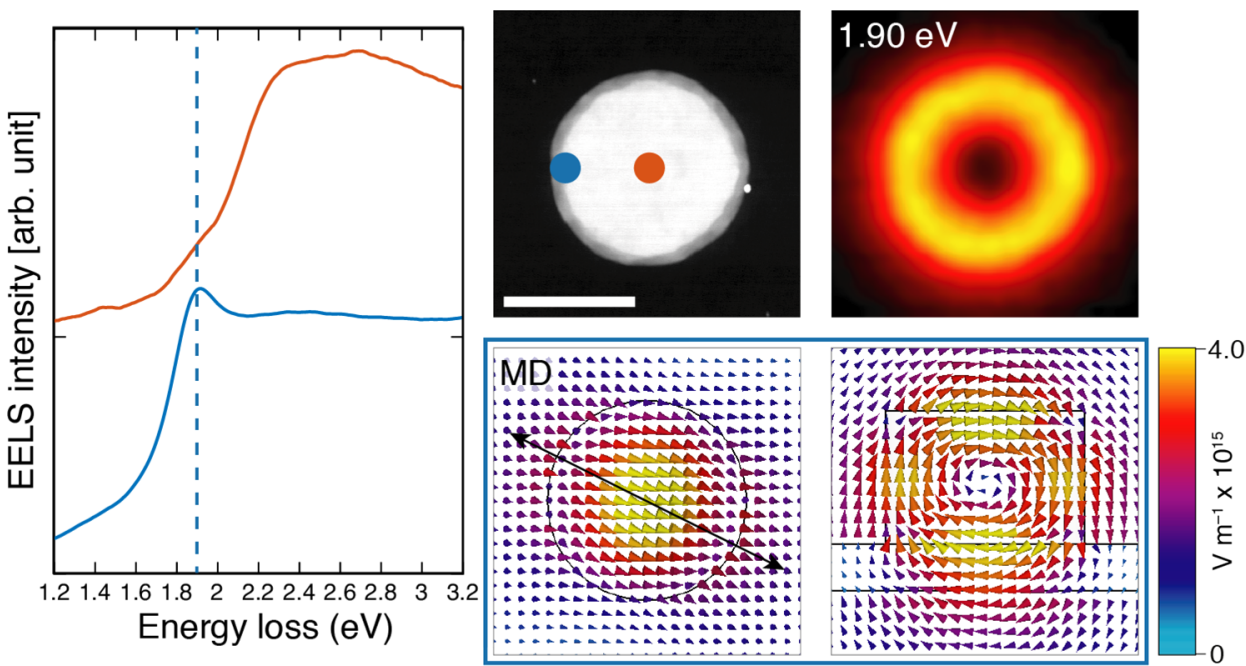

Figure 4. Analysis of the edge excitation of the magnetic dipole (MD) in a $150 \mathrm{~nm}$ disc. The left panel shows EEL spectra integrated from rings at different radii, as indicated using color-coded spots on the HAADF STEM image in the middle-top panel. The $1.90 \mathrm{eV}$ EELS heat intensity map is from an energy window centered on the peak marked on the blue EEL spectrum from the disc edge. It corresponds to the excitation of a MD eigenmode, whose simulated electric field distribution is shown below in plan-view (left) and cross-section (right). The plan-view distribution shows the chord along which the cross-section cut-plane is taken. Complementary EELS data are shown in SI Figure S1-1. The color bar shows the scale for the electric field maps. Scale bar: $100 \mathrm{~nm}$.

nanocavities privileges the excitation of eigenmodes that have vertically oriented current loops which meet at one or more loci within the nanocavity. In comparison, impacting the electron probe at or near the nanocavity edge changes the excitation geometry, such that many other eigenmodes can instead be excited. In principle, the lowest-energy modes that can be edge-excited are either the in-plane $\mathrm{MD}$, as stimulated by the electron probe pushing away charge at the entry or exit surfaces of the nanocavity, or the in-plane ED, as stimulated by the probe pushing away charge at any point of its trajectory. For dielectric discs of fixed height $h$, the respective resonance energies of these two modes can be tuned independently by changing the disc diameter $d$. Smaller, more equiaxed discs tend to the spherical case, for which the MD has the lower energy. ${ }^{62}$ However, when $d \gg h$, while the wavelength and energy of the in-plane polarized ED scale primarily with $d$, the current loop of the MD is geometrically constrained by $h$. As a result, the ED red-shifts more strongly than the $\mathrm{MD}$, leading to a crossover in their resonance energies. ${ }^{33-35,63}$

From the above, EEL spectra recorded from the edges of small discs are expected to show a lowest energy peak that corresponds to the excitation of the MD (also known as $\left.\mathrm{HEM}_{11 \delta}\right) .{ }^{57}$ As an example, Figure 4 shows data from a $150 \mathrm{~nm}$ disc. The blue EEL spectrum from the disc edge shows a distinct peak at $1.90 \mathrm{eV}$, whose strong edge excitation is confirmed by the accompanying EELS intensity map. In accord with the hypothesis, the peak energy matches closely to the $1.86 \mathrm{eV}$ simulated resonance energy of the MD shown below the map, in contrast to the ED at $\sim 2.09 \mathrm{eV}$. Further supporting the analysis, frequency domain simulations of disc excitation using edge-placed dipoles (both vertical and horizontal in order to represent different aspects of the evanescent field of the electron probe) uniquely favor the MD over the ED (see SI Figure S3-1).

As the size of the discs is increased, simulations imply that the crossover for the lowest-energy dipole mode changing from $\mathrm{MD}$ to ED occurs at a diameter of $\sim 275 \mathrm{~nm}$. Therefore, considering the $450 \mathrm{~nm}$ disc, we could expect that the lowest- energy peak for the edge-located EEL spectrum corresponds to the excitation of an $\mathrm{ED}$, as seen at $\sim 1.00 \mathrm{eV}$ on the dark blue curve in Figure 1. The accompanying EELS map in Figure 5
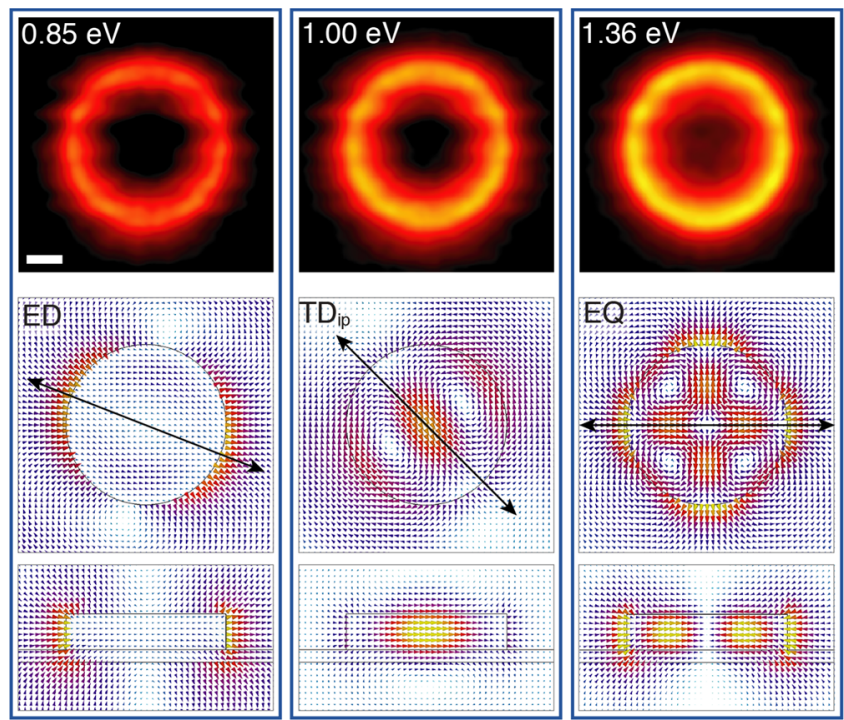

Figure 5. Edge-excited resonances for a $450 \mathrm{~nm}$ a-Si disc. The top row shows EELS maps for the indicated energy losses. The EELS response of the $0.85 \mathrm{eV}$ map is primarily driven by the excitation of the electric dipole (ED) eigenmode, whose electric field distribution is shown below it. Going to higher energy in the same extended spectral peak, the excitation is hypothesized to become more in-plane toroidal dipole $\left(\mathrm{TD}_{\mathrm{ip}}\right)$ in character, whose electric field nature is shown below the $1.00 \mathrm{eV}$ map. The $1.36 \mathrm{eV}$ EELS map corresponds to a narrower EELS peak, as seen on the dark blue spectrum in Figure 1. This derives from the excitation of the electric quadrupole (EQ) eigenmode shown below it. The plan-view field distributions show the chords along which the cross-section cut-planes are taken. For the color scale of the electric field maps, the reader is referred to Figure 2. Scale bar: $100 \mathrm{~nm}$. 


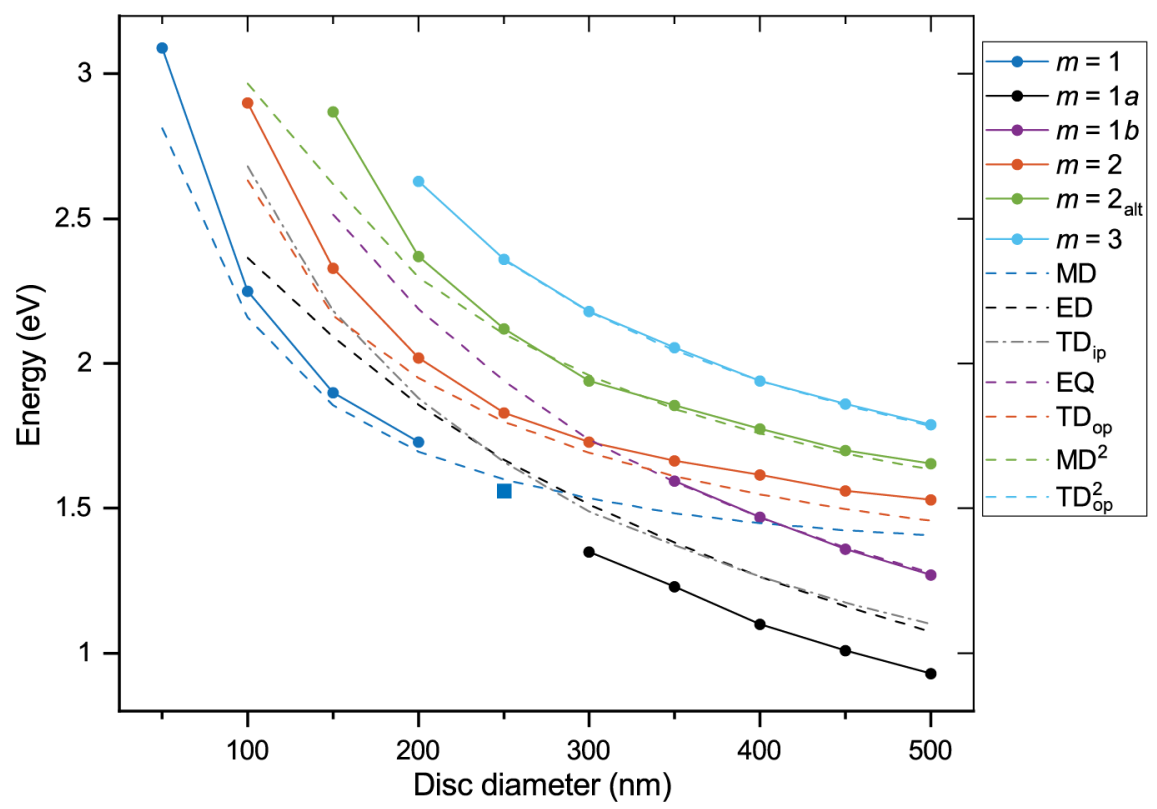

Figure 6. Experimental and theoretical dispersion curves for excitations in 50-500 nm diameter a-Si discs of $100 \mathrm{~nm}$ thickness on $35 \mathrm{~nm}$ thick $\mathrm{Si}_{3} \mathrm{~N}_{4}$ membranes. The solid lines show the experimental curves extracted from EELS peaks identified for different excitations from their STEM-EELS map data cubes, where $m$ is the number of nodes in a map. The dashed lines correspond to the resonance energies for the following eigenmodes simulated using CST Studio Suite: magnetic dipole (MD); electric dipole (ED); in-plane toroidal dipole (TD ${ }_{\text {ip }}$ ); electric quadrupole (EQ); out-of-plane toroidal dipole $\left(\mathrm{TD}_{\mathrm{op}}\right)$; second-order magnetic dipole $\left(\mathrm{MD}^{2}\right)$ and second-order out-of-plane toroidal dipole $\left(\mathrm{TD}_{\mathrm{op}}^{2}\right)$. Color-coding is used to indicate correspondences between experimental and theoretical curves, and relates to that used for the EEL spectra in Figures 1 and 4. The dark blue square is for the edge excitation EELS peak of the $250 \mathrm{~nm}$ disc, which sits at the crossover between the MD and ED/TD ${ }_{\text {ip }}$ excitation curves. (To help comparison with other works, SI Figure S1-11 shows the same data replotted with respect to the optical wavelength of an excitation.)

confirms that this excitation is localized to the disc edge, while the ED panel shows the electric field nature of this excitation, which is also termed $\mathrm{TE}_{111}{ }^{41}$ To fully correspond the experiment and simulation, however, various factors must be considered. First, the EELS peak has a broad, extended shape; quite different to that for excitation of the MD in the $150 \mathrm{~nm}$ disc. Indeed, the excitation is already visible in the $0.85 \mathrm{eV}$ EELS intensity map shown in Figure 5. Second, the $1.00 \mathrm{eV}$ peak energy is strongly red-shifted compared with the simulated ED resonance energy of $1.16 \mathrm{eV}$. Finally, an inplane toroidal dipole $\left(\mathrm{TD}_{\mathrm{ip}}\right)$, having a very similar resonance energy of $1.17 \mathrm{eV}$, could also be excited by the electron probe at the disc edge. Having an electric field nature shown in the $\mathrm{TD}_{\text {ip }}$ panel of Figure 5, this eigenmode is also known as $\mathrm{ED}^{2}$ or $\mathrm{HEM}_{12 \delta} \cdot{ }^{50,57}$ We believe that the observed EELS signal actually results from a superposition of both the $\mathrm{ED}$ and $\mathrm{TD}_{\mathrm{ip}}$ excitations but with each making a different contribution. In dielectric discs, the ED's electric field is poorly confined within the nanocavity volume, as seen in Figure 5. In consequence, it is a broad resonance having a very low $Q$-factor, ${ }^{34,41}$ with a simulated value of just $\sim 1.5$ for the $450 \mathrm{~nm}$ disc (as compared with $\sim 12$ for the $\mathrm{MD}$ of the $150 \mathrm{~nm}$ disc). As known from plasmon EELS, the increased damping of a low Q-factor mode leads to the broadening of its EELS peak. ${ }^{27}$ This broadening is very pronounced for this ED eigenmode. The low $Q$-factor of the ED may also be responsible for the observed red-shift effect, since such a lossy resonance will be optimally driven at a frequency different to its resonant frequency. In contrast, the $\mathrm{TD}_{\text {ip }}$ is a narrower resonance, with a simulated $Q$-factor of $\sim 7$. We therefore hypothesize that, far below the $\mathrm{ED} / \mathrm{TD}_{\text {ip }}$ resonance energy, the electron probe stimulates an excitation which is primarily ED in character. As the "ideal" resonance energy is approached, the excitation becomes progressively $\mathrm{TD}_{\text {ip }}$-like in character. Visual inspection of simulated near-field maps of the disc excited by a dipole at different frequencies suggest such a progression (see SI Figure S3-2). Nevertheless, while we later validate the dual excitation of these eigenmodes with the study of the ellipse and rectangle, a complete multipole decomposition of the simulated field distributions would be needed to confirm the hypothesized modal progression. ${ }^{63}$ This EELS response can also be related to the scattering of planewave EM illumination by the disc. In the latter case, the anapole is produced when a negative contribution to extinction from the $\mathrm{TD}_{\text {ip }}$ cancels radiation from the ED, giving a strong dip in the far-field scattering (see simulations in SI Figure S4-1). ${ }^{40-42}$

The results point to another contrast between the EELS and light-optical response. The ability of planewave EM radiation to excite both the ED and MD in a single disc is key to certain optical tuning strategies. ${ }^{35,41}$ However, with EELS, we observe only an $\mathrm{MD}$ peak in smaller discs and only an $\mathrm{ED} / \mathrm{TD}_{\text {ip }}$ peak in larger discs. In Figure 6, this behavior is studied with dispersion curves for the different EELS peaks of discs varying in diameter from $50-500 \mathrm{~nm}$. The plots for the lowest energy edge-excited modes are split into two curves, each having a single node $m$ in the intensity map for the EELS peak. The first $m=1$ curve derives from the smaller discs. It clearly conforms to the dispersion curve for the simulated $\mathrm{MD}$ resonance energies, with a consistent blue shift between the simulated and experimental values (as seen for the $150 \mathrm{~nm}$ disc above). The second, $m=1 a$ curve, derives from the larger discs. Its form tends toward that of the steeper $\mathrm{ED} / \mathrm{TD}_{\mathrm{ip}}$ curves, rather than the flattening MD one, ${ }^{34}$ helping confirm its assignment to these eigenmodes. As discussed for the $450 \mathrm{~nm}$ disc, it 

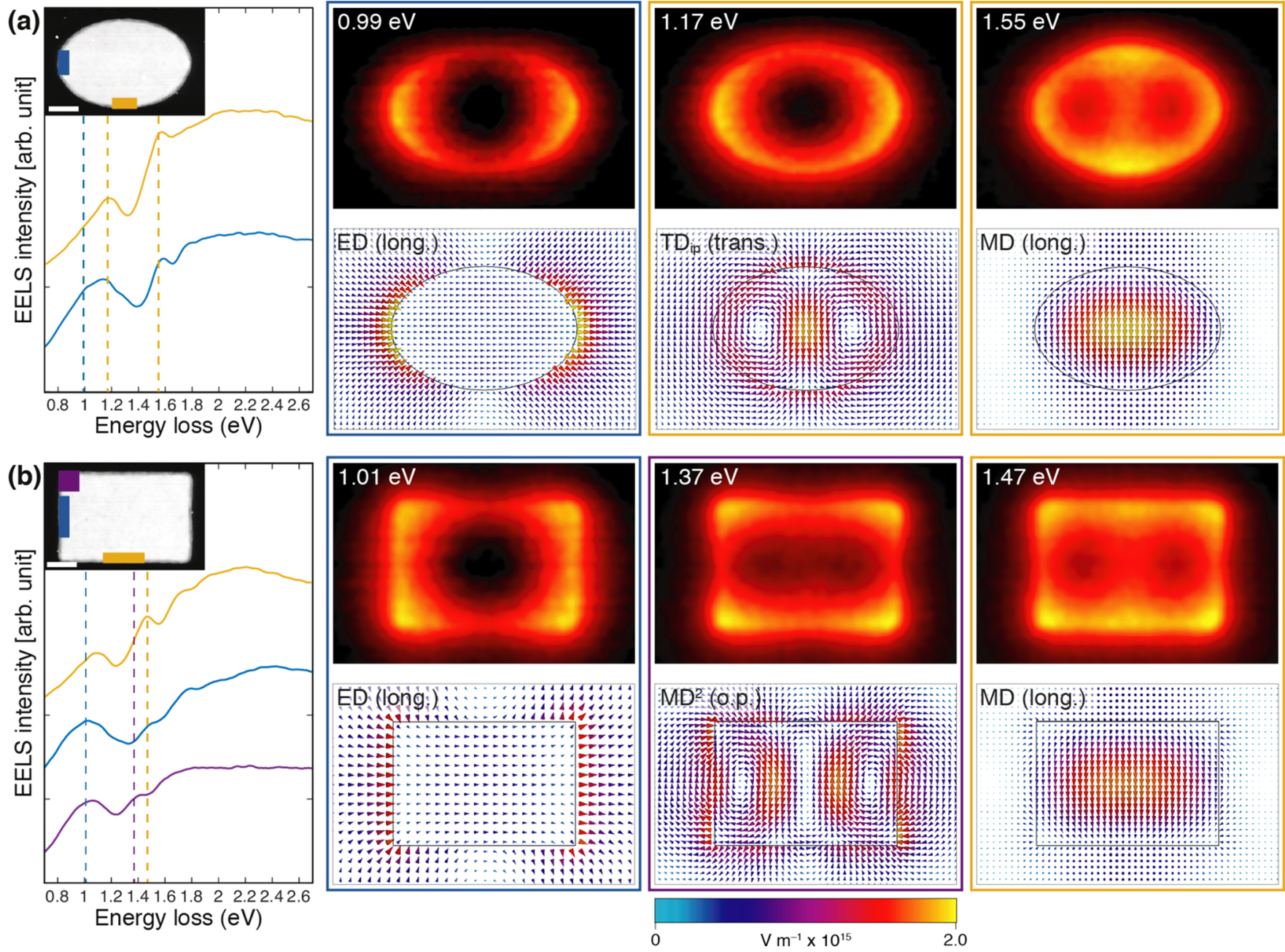

Figure 7. Analysis of edge-excited resonances in a $450 \times 300 \mathrm{~nm}$ (a) ellipse and (b) rectangle. The EEL spectra are integrated from the impact parameters illustrated with color-coded boxes on the respective HAADF STEM images. In part (a) for the ellipse, the 0.99, 1.17, and $1.55 \mathrm{eV}$ EELS maps are, respectively, related to the electric field distributions of the longitudinal ED, transverse $\mathrm{TD}_{\mathrm{ip}}$, and longitudinal $\mathrm{MD}$ eigenmodes (shown in plan-view). The 1.01, 1.37, and 1.47 eV EELS maps for the rectangle in part (b) are, respectively, corresponded to the longitudinal ED, out-of-plane $\mathrm{MD}^{2}$ and longitudinal $\mathrm{MD}$ eigenmodes. SI Figures S1-4/S1-5 and S2-4/S2-5 present complementary experimental and simulation data, while the color bar gives the scale for the electric field maps. Scale bars: $100 \mathrm{~nm}$.

consistently displays a red shift from simulated to experimental values. The crossover in excitation nature from $\mathrm{MD}$ to $\mathrm{ED} /$ $\mathrm{TD}_{\text {ip }}$ happens at around $250 \mathrm{~nm}$ in disc diameter. With the EELS data point for this disc sitting between the two curves, as shown with a dark blue square in Figure 6, both its EELS peak shape and preliminary simulations suggest that it derives from a dual excitation of the $\mathrm{MD}$ and $\mathrm{ED} / \mathrm{TD}_{\mathrm{ip}}$. Aside from this exception, the evolution of EELS excitation probability from favoring the $\mathrm{MD}$ below the cross-cover, to $\mathrm{ED} / \mathrm{TD}_{\text {ip }}$ above it, is confirmed by the previously discussed frequency domain dipole excitation simulations. While this difference compared to the planewave response must derive from a difference in the nature of the electric field driving the excitation-localized for the electron source as opposed to extended for EM illumination-it would be interesting to pursue a deeper physical understanding of the phenomenon in the future.

The dispersion curves help to identify another mode. For discs $\geq 350 \mathrm{~nm}$ in diameter, an additional, higher-energy edgeexcited EELS peak is present, such as the $1.36 \mathrm{eV}$ peak seen on the dark blue curve in Figure 1 for the $450 \mathrm{~nm}$ disc. In Figure 6 , the dispersion curve for this peak is labeled the $m=1 b$ curve. It bears a near-perfect match $( \pm 0.01 \mathrm{eV})$ to that of an electric quadrupole (EQ) mode. The electric field nature of this mode is illustrated in Figure 5, to compare to the $1.36 \mathrm{eV}$ EELS intensity map shown in the panel above it. Compared with the $\mathrm{ED} / \mathrm{TD}_{\mathrm{ip}}$ excitation, the EQ gives a much sharper EELS peak. This correlates well with the $Q$-factor of the mode being an order of magnitude higher than for the ED, with a simulated value of $\sim 20$. The much lower damping of the eigenmode likely also explains the close correspondence of EELS peak and predicted eigenmode resonance energies. Frequency domain simulations give further support to this assignment (see SI Figure S3-2). The same simulations also predict the sharp excitation of a $1.58 \mathrm{eV}$ electric hexapole mode, which is indeed observed as a small $1.60 \mathrm{eV}$ peak in the edge EEL spectrum of the $450 \mathrm{~nm}$ disc in Figure 1. Assignment of another peak at $1.72 \mathrm{eV}$ is less definitive, but it could derive from an off-resonance excitation of the $\mathrm{MD}^{2}$, perhaps mixed with some electric octupole character. Finally, we note that, with the electric field vectors of the $\mathrm{ED}, \mathrm{TD}_{\mathrm{ip}}$, and EQ being primarily in the plane of the nanocavity, it might be surprising that they induce an energy loss on the electron propagating 
normal to the plane. The observed energy loss could be from the residual out of plane field components seen, for instance, in the cross-section field distribution maps of Figure 5, or from extra field components that might be induced by the transmitting point charge.

In Figure 6, dispersion curves for the internally excited $\mathrm{TD}_{\text {op }}$, $\mathrm{MD}^{2}$ and $\mathrm{TD}_{\mathrm{op}}^{2}$ eigenmodes are also plotted. The EELS curves for exciting the $\mathrm{TD}_{\mathrm{op}}$ and $\mathrm{MD}^{2}$ are respectively labeled as $m=$ 2 and $m=2_{\text {alt }}$, since both have two nodes in intensity maps but excitation of the $\mathrm{MD}^{2}$ gives an alternative intensity distribution to exciting the $\mathrm{TD}_{\mathrm{op}}$. The $m=2_{\text {alt }}$ curve and the $m=3$ curve for exciting the $\mathrm{TD}_{\mathrm{op}}^{2}$ both adhere closely to their simulated eigenmode counterparts. Further, the previously discussed blue shift of the $m=2$ EELS peak compared with eigenmode resonance energy for the $\mathrm{TD}_{\mathrm{op}}$ is confirmed across the diameter range.

Edge Excited Eigenmodes: Ellipse, Rectangle, Triangle, and Square. Having identified eigenmodes that are edge-excited in the discs, we now study how they are modulated by changing the nanocavity shape, starting with the ellipse. By breaking degeneracy, the ellipse separates the $\mathrm{ED}$ and $\mathrm{TD}_{\text {ip }}$ eigenmodes into orthogonal lower-energy and higher-energy branches. Out of these, the lower-energy ED eigenmode is longitudinal in nature, as illustrated for a $450 \times$ $300 \mathrm{~nm}$ ellipse in Figure $7 \mathrm{a}$, where it has a predicted resonance energy of $1.17 \mathrm{eV}$ and a $Q$-factor of $\sim 2$. Considering its electric field distribution, the probability of exciting this mode will be highest when the electron probe impacts at the longitudinal ends of the ellipse. In contrast, the elliptical geometry constrains the lower-energy $\mathrm{TD}_{\mathrm{ip}}$ to a form of two longitudinally arranged current loops. This eigenmode's dipole lies on a transverse axis, as shown on the $\mathrm{TD}_{\mathrm{ip}}$ (trans.) panel of Figure $7 \mathrm{a}$, such that it will instead be excited by a probe impacting near the transverse edges of the ellipse. It also has a higher predicted resonance energy of $1.26 \mathrm{eV}$, and a greater $Q-$ factor of $\sim 10$. The different nature of these two eigenmodes allows us to separate their excitations in energy and space. Specifically, the blue EEL spectrum in Figure 7a from the longitudinal end of the ellipse shows a lower peak energy of $\sim 1.13 \mathrm{eV}$. The peak is broad, with significant intensity at lower energies forming a hump at $\sim 0.99 \mathrm{eV}$. These traits correlate well to the excitation of the low Q-factor longitudinal ED. In geometric agreement, the $0.99 \mathrm{eV}$ EELS map shows notably strong excitation at the longitudinal ends of the ellipse. In comparison, the orange spectral curve in Figure $7 \mathrm{a}$ from the transverse edge of the ellipse shows both a higher peak energy of $\sim 1.17 \mathrm{eV}$, and markedly lower intensities below the peak energy, in keeping with expectations for the unique excitation of the transverse $\mathrm{TD}_{\mathrm{ip}}$. Tied to this, the $1.17 \mathrm{eV}$ EELS map in Figure $7 \mathrm{a}$ shows an enhanced intensity at the transverse edges of the ellipse. The increased aspect ratio of a $600 \times 300 \mathrm{~nm}$ ellipse leads to an even greater separation of the two eigenmodes, with the $\mathrm{ED}$ and $\mathrm{TD}_{\text {ip }}$ EELS spectra peaking at 0.97 and $1.10 \mathrm{eV}$, respectively (see SI Figure S1-6). In comparison, excitation of the high-energy branches of the ED and $\mathrm{TD}_{\text {ip }}$ is not obvious in the STEM-EELS data. This differs from how such shapes would interact with planewave light, where illumination polarized parallel to the longitudinal axis is expected to excite the longitudinal $\mathrm{ED}$ and $\mathrm{TD}_{\mathrm{ip}}$ eigenmodes, and transverse polarization the transverse ones, leading to different far-field scattered power for the two polarization axes (e.g., see SI Figure S4-2).
The $450 \times 300 \mathrm{~nm}$ rectangular nanocavity displays similar EELS characteristics for longitudinal ED and transverse $\mathrm{TD}_{\mathrm{ip}}$ excitations, as seen by comparing the blue longitudinal end and orange transverse edge spectra in Figure $7 \mathrm{~b}$. It further allows us to explore differences between the EELS response of planar plasmonic and dielectric resonators. In the former, the EELS intensity patterns observed along the resonator sides correspond to edge modes, which are quasi one-dimensional resonances. ${ }^{4-6,17}$ For straight-edged structures, these can be thought of as standing wave patterns along the edges, similar to those obtained for plasmonic rods, for which the electron probe excites the resonance at the nodes of the standing wave. In the dielectric resonators, the edge-placed electron probe instead excites resonances within the cavity, which extend through its volume. Considering the rectangle, its longitudinal ED mode shows a roughly uniform electric field distribution along the longitudinal edges, see Figure $7 \mathrm{~b}$. Therefore, the probability of exciting this mode is approximately constant along the length of the edge. In correspondence, the $1.01 \mathrm{eV}$ EELS map of Figure $7 \mathrm{~b}$ for the ED excitation shows an intensity which is basically uniform along the length of the rectangle's short edge-very different to the nodal pattern expected for a dipole edge resonance in a plasmonic system. The same phenomenon is observed for the longitudinal ED resonances of larger $600 \times 300 \mathrm{~nm}$ and a $1000 \times 500 \mathrm{~nm}$ rectangles (SI Figures $\mathrm{S} 1-7$ and $\mathrm{S} 1-8$ ).

Going up in energy loss, the $450 \times 300 \mathrm{~nm}$ rectangle shows another characteristic excitation, which is again markedly different to those seen in plasmonic systems. Shown with the $1.37 \mathrm{eV}$ map in Figure $7 \mathrm{~b}$, it has an EELS intensity which is strongest at the corners but that also extends along the edges, in particular the long ones. This EELS response corresponds to the excitation of an $\mathrm{MD}^{2}$ eigenmode which, as illustrated in the $\mathrm{MD}^{2}$ (o.p.) panel in the figure, is out-of-plane, as opposed to the in-plane $\mathrm{MD}^{2}$ eigenmode excited within the volume of the rectangle. With a closely matched $1.41 \mathrm{eV}$ predicted resonance energy, dipole simulations confirm the excitation of this mode at the rectangle's corners and away from the corners moving along the long edge toward (but not at) their midpoints (see SI Figure S3-5). They further suggest that the actual excitation will be somewhat transient, with the current loops traveling across the volume of the rectangle (SI Figure S3-6). The $600 \times$ $300 \mathrm{~nm}$ rectangle shows the same type of EELS response at $\sim 1.19 \mathrm{eV}$, indicating some shape generality to this excitation (see SI Figure S1-7).

While the ellipse cannot show such a corner mode, both the ellipse and rectangle share a different feature in common: a strong excitation at the midpoint of the transverse edges, at similar energy losses for similar nanocavity dimensions. This is seen in the 1.55 and $1.47 \mathrm{eV}$ maps for the $450 \times 300 \mathrm{~nm}$ ellipse and rectangle in panels a and b, respectively, of Figure 7. This EELS response probably derives from the excitation of longitudinal MD modes, as illustrated for both forms in Figure 7. Not only does this assignment fit the intensity distribution in the EELS maps, but further support is provided by the EELS peak energies having a slight blue shift compared to the predicted resonance energies ( 1.48 and $1.45 \mathrm{eV}$ for the ellipse and rectangle, respectively) - as was also found for the $\mathrm{MD}$ response in the small discs. Finally, simulations with a dipole placed at the midtransverse edge location of the rectangle also show a strong MD response (see SI Figure S3-5). Since the transverse dimension of $300 \mathrm{~nm}$ is larger than the MD/ED crossover diameter of the discs, it appears that deviating from 

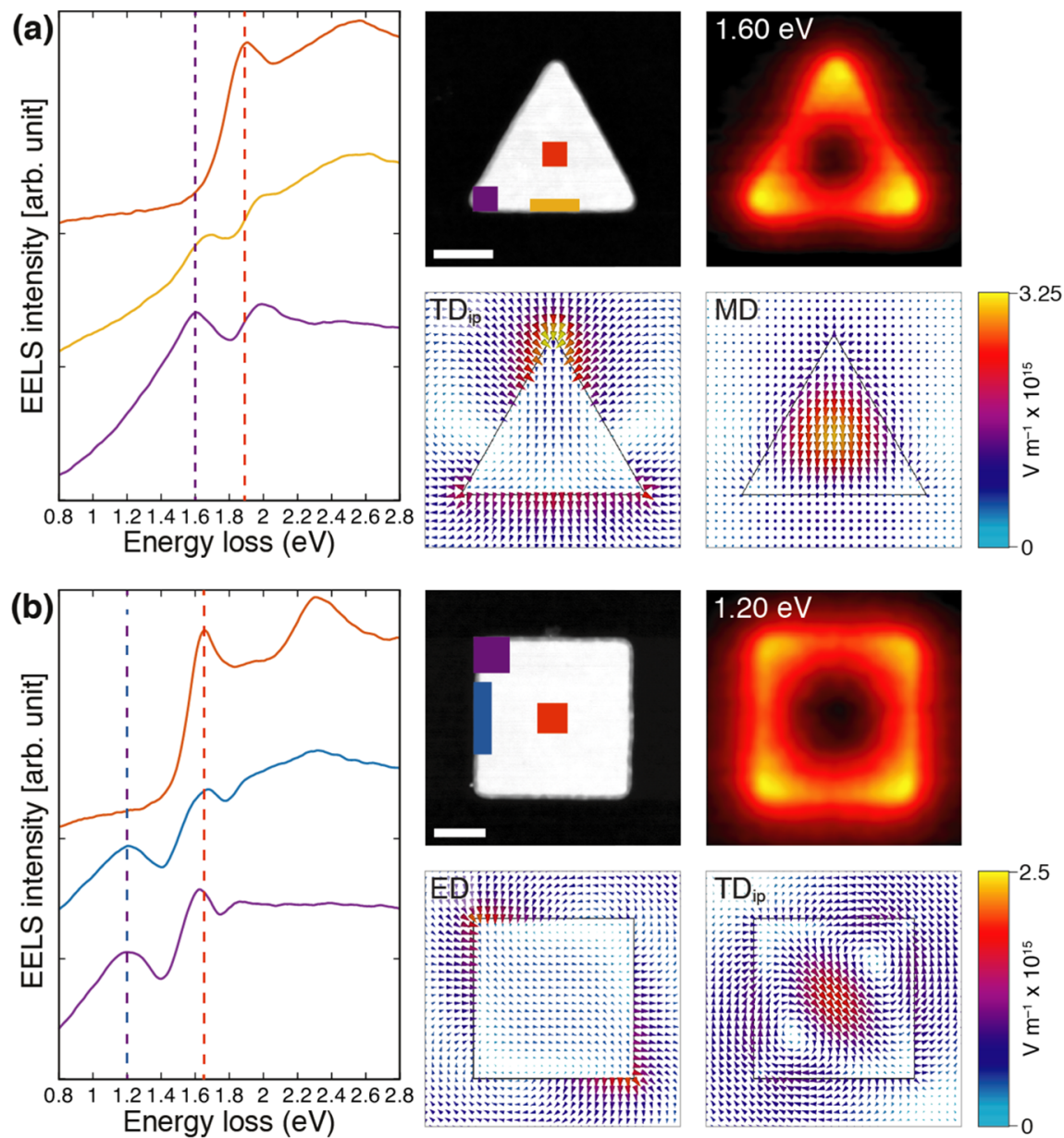

Figure 8. Analysis of corner- and edge-excited resonances in a $300 \mathrm{~nm}$ side (a) triangle and (b) square. The EEL spectra in the left panels are integrated from the impact parameters illustrated with color-coded boxes on the HAADF STEM images in the middle-top panels. In part (a), the $1.60 \mathrm{eV}$ map corresponds to the peak on the purple EEL spectrum from the triangle's corner. This is related to the $\mathrm{TD}_{\text {ip }}$ eigenmode depicted below-left, while the MD shown below-right is responsible for the higher-energy peak at $\sim 1.66 \mathrm{eV}$ on the orange spectral curve

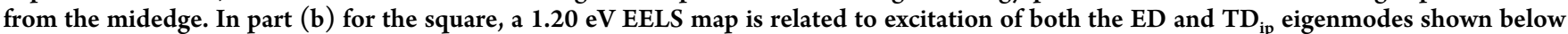
it. The electric field distributions are shown in plan-view, with color bars giving their respective scales. EEL spectra from the nanocavity centers are also shown with red curves, with red vertical dashed lines indicating the TD $_{\text {op }}$ peaks mapped in Figure 3a. SI Figures S2-2 and S23 present complementary simulated eigenmode views. Scale bars: $100 \mathrm{~nm}$.

an aspect ratio of one plays a role in favoring the $\mathrm{MD}$ excitation here. Indeed, similar EELS phenomena are observed for the $600 \times 300 \mathrm{~nm}$ ellipse and rectangle (SI Figures S1-6 and S1-7).

Unlike the rectangle, the triangle shows a lowest-energy edge excitation that is highly localized to its corners, as shown in the $1.60 \mathrm{eV}$ intensity map of Figure 8a. Furthermore, the corresponding peak on the purple EEL spectrum is relatively sharp. The narrow vertex of the triangular form is incompatible with the extended electric field distribution of the ED, such that this eigenmode is suppressed. Instead, only a $\mathrm{TD}_{\mathrm{ip}}$ eigenmode is excited, explaining the sharp EELS peak from the sole excitation of this narrower resonance. Further, the electric field map of the triangle's $\mathrm{TD}_{\text {ip }}$ in Figure $8 \mathrm{a}$ presents poloidal electric field loops that are not fully contained within the body of the nanocavity. Tied to this, the eigenmode is geometrically fixed to the axis of the triangle, and can therefore only be strongly excited at the corners. Even if the resultant EELS map looks similar to that for the corner-excited dipole resonance of plasmonic nanotriangles, its underlying nature is strongly different. ${ }^{1,6,17}$ In correlation to the lack of an ED, farfield scattering simulations with planewave EM illumination show a weak scattered power at lower energies, with no anapole dip near its $\mathrm{TD}_{\text {ip }}$ resonance energy (see SI Figure S4$1)$.

At the midedge impact parameter on the triangle, there is a higher-energy EELS peak at $\sim 1.66 \mathrm{eV}$, as seen on the orange spectral curve in Figure 8a. This derives from the excitation of a $\mathrm{MD}$ having a correspondingly higher predicted resonance energy, as also illustrated in Figure 8a. The flip from exciting a $\mathrm{TD}_{\text {ip }}$ at the corner, to a $\mathrm{MD}$ at the opposite side, is confirmed by frequency domain simulations (SI Figure S3-4). This interplay relates to the triangle's size, it having an equivalent circle diameter of $\sim 250 \mathrm{~nm}$ that is near the MD/ED crossover size for the discs. At yet higher-energy losses, it appears that a magnetic hexapole is excited at both corners and midedge, producing the $\sim 1.99 \mathrm{eV}$ peak in the corner and midedge spectra of Figure 8 a.

As with the triangle, the $300 \mathrm{~nm}$ square shows enhanced excitation at its corners for its lowest-energy excitation, see the $1.20 \mathrm{eV}$ map of Figure $8 \mathrm{~b}$. Nevertheless, there are some key differences. Unlike the triangle, the edge and corner spectra in Figure $8 \mathrm{~b}$ both show the same form for the lowest-energy peak, just with the blue edge spectrum being less intense than the 
purple corner one. The peak also has a left-biased hump-like shape, indicative of dual $\mathrm{ED} / \mathrm{TD}_{\mathrm{ip}}$ excitation. Indeed, both such eigenmodes are valid for the square, as illustrated in their respective panels in Figure $8 \mathrm{~b}$. Like for the discs, the EELS peak is strongly red-shifted relative to their predicted resonance energies of $\sim 1.40 \mathrm{eV}$. Further, unlike the $\mathrm{TD}_{\text {ip }}$ of the triangle, these modes are unconstrained and free to rotate around the axis of the square, hence the observation of their excitation at both corners and edges. Experimentally, however, the excitation probability at the corner is enhanced relative to the midedge location. In agreement with the unconstrained orientations of the two eigenmodes, simulations indicate that the far-field scattering of planewave EM illumination should be very similar whether it is polarized parallel to the square's edge or diagonal (see SI Figure S4-1).

At higher-energy losses, the purple EEL spectrum in Figure $8 \mathrm{~b}$ from the square's corner shows a distinct peak at $1.62 \mathrm{eV}$. This is ascribed to the excitation of a diagonal EQ which, as for the EQ eigenmodes of the discs, has a closely matched predicted resonance energy of $1.61 \mathrm{eV}$. The EQ eigenmode solution is degenerated into this diagonal variant and a transverse one (see SI Figure S2-3), the latter having a lower predicted resonance energy of $1.59 \mathrm{eV}$. The transverse EQ should instead be excited by an electron probe impacting at the midedge location. Indeed, its signature is observed as a bump at $\sim 1.58 \mathrm{eV}$ on the blue EEL spectrum from the square's edge in Figure 8b. Observing this small shift between the two excitations emphasizes the spatial-spectral sensitivity of the STEM-EELS technique. The result also demonstrates how deviations from a disc shape can be used to tailor the eigenmodes in subtle but specific ways.

\section{DISCUSSION}

Overall, the presented analyses prove the sensitivity of EELS mapping for probing the eigenmodes supported by dielectric nanocavities, with many displaying a nanometric spatial agreement between experiment and theoretical expectations. One of the finest details resolved in the data is the $\mathrm{TD}_{\mathrm{op}}^{2}$ eigenmode, visible in discs down to $250 \mathrm{~nm}$ in diameter. Measurement of its intensity profile from, for instance, a 300 $\mathrm{nm}$ disc (see SI Figure S1-2), suggests an ultimate "spatial definition" for the technique of $\sim 30 \mathrm{~nm}$. Nevertheless, the spatial extent of an excitation is contingent on its nature. For instance, in discs, simulations suggest that the electron probe still excites the $\mathrm{TD}_{\mathrm{op}}$ at the radius at which the $\mathrm{MD}^{2}$ is optimally excited (see SI Figure S3-3). In agreement, the spectra in Figure 1 and map in Figure 2 show that the $1.56 \mathrm{eV}$ peak for the $\mathrm{TD}_{\text {op }}$ excitation in the $450 \mathrm{~nm}$ disc has a very wide spatial distribution. However, the reverse is not true, since the electric field of the $\mathrm{MD}^{2}$ is fundamentally incompatible with the field of the probe at the disc center. As a result, the $\mathrm{MD}^{2}$ eigenmode has a narrower intensity distribution in its $1.70 \mathrm{eV}$ EELS map in Figure 2, with the $1.87 \mathrm{eV}$ map of the $\mathrm{TD}_{o p}^{2}$ eigenmode being sharper still. In the opposite direction, the most spatially extended features in the data belong to a featureless accumulation of EELS intensity in the center of the resonators that correlates to very broad energy-loss peaks centered on energies of $\sim 2.25-2.35 \mathrm{eV}$, as can be seen on the red EELS curves of Figures 1 and 8 (and in the SI STEMEELS Data section). With moderate but also inconsistent redshifts with increasing nanocavity size, the origin of this is yet to be determined but perhaps could relate to a type of "breathing mode". 3,6,64
In order to identify the influence of the $\mathrm{Si}_{3} \mathrm{~N}_{4}$ support membrane on the results, eigenmodes have been simulated for freestanding nanocavities. The results indicate that the membrane introduces minor red-shifts of $\sim 0.015-0.08 \mathrm{eV}$ in resonance energies, and moderate reduction of $Q$-factors. These changes derive from electric field leakage into the membrane, such that it forms an extension of the resonator. ${ }^{34}$ Consistent with this, the red-shift is more pronounced for eigenmodes having vertically circulating displacement current loops, via effective increase of the resonator depth, or being poorly confined $\left(\mathrm{ED}, \mathrm{MD}, \mathrm{TD}_{\mathrm{op}}, \mathrm{MD}^{2}\right.$ : red-shifts $\sim 0.05-0.08$ $\mathrm{eV}$ ), than for modes whose polarization is primarily confined within the in-plane dimension $\left(\mathrm{TD}_{\mathrm{ip}}, \mathrm{EQ} \mathrm{EH}\right.$ : red-shifts $\sim 0.015-0.03 \mathrm{eV}$ ). In comparison, similar support membranes in planar contact with plasmonic structures can produce a much stronger effect on LSPR energies, from resonance hybridization at the interface. ${ }^{54,55}$ The nanocavity volume effect may also be (in part) responsible for the blue shifts of $\mathrm{MD}, \mathrm{TD}_{\mathrm{op}}$, and $\mathrm{MD}^{2}$ EELS peak energies, relative to their simulated resonance counterparts, that is seen as nanocavity size reduces (e.g., see Figure 6), from a slight tapering in of the upper parts of the nanocavity walls reducing their crosssectional area as compared to the straight-sided walls used in the simulations (see SI).

The Eigenmode Solver analysis, backed up by frequency domain dipole excitation and far-field scattering simulations, proves to be an effective tool for interpreting the experimental data and connecting to light-optical properties. While the frequency domain simulations present features that can be compared to EELS peaks, further adaptation of our approach is needed to compute the complete loss function, as is possible for the surface-bound excitations of plasmonics using, for instance, the boundary element method. ${ }^{26}$ Toward this goal, Kordahl et al. have developed an electrodynamic framework for simulating the EELS response for fast electrons penetrating dielectrics with a disc geometry, using an analytical waveguide approach. $^{65}$ Their method shows promise, even if the discrepancy between experimental peak and simulated excitation energies is greater than for the analyses made here. Finally, as with studies of plasmonics, the radially symmetric field of the STEM-EELS probe is "blind" to certain types of excitations. Exploiting the quantum nature of the interaction of the electron with a nanocavity photonic excitation using phase-shaped probes could be a strategy for accessing other eigenmodes. 29,30

\section{CONCLUSIONS}

EELS mapping has been used to investigate the EM resonances excited in geometrically controlled planar dielectric nanocavities analogous to those used in photonic applications, with results correlated to eigenmodes identified by simulation. Unlike for plasmonic systems, edge- and corner-stimulated excitations correspond to cavity modes. For smaller discs, edge-placed probes excite the $\mathrm{MD}$, flipping to a superposed $\mathrm{ED} / \mathrm{TD}_{\text {ip }}$ excitation at larger sizes, indicating a change in excitation probability that deserves further exploration. The repartition between the broad $\mathrm{ED}$ and narrow $\mathrm{TD}_{\text {ip }}$ resonances can be tuned using the nanocavity shape, for instance suppressing the ED with a triangle or separating the responses in space and energy with an ellipse. Different to the typical dipole response in plasmonic systems, the ED response is extended, both spatially and energetically. At higher-energy losses, other cavity modes such as the out-of-plane $\mathrm{MD}^{2}$ and 
EQ are also observed at the edges. Placing the electron probe further within the nanocavity instead privileges the excitation of resonances having vertically circulating current loops that meet in the volume, such as the $\mathrm{TD}_{\mathrm{op}}$ and in-plane $\mathrm{MD}^{2}$ eigenmodes. While the signature of the nondegenerate $\mathrm{TD}_{\text {op }}$ changes little with nanocavity form, the $\mathrm{MD}^{2}$ eigenmodes are constrained by shape, producing symmetry-related patterns of intensity hotspots in the corresponding EELS maps for ellipse, rectangle, triangle and square nanocavities. Overall, the results show the potential of the STEM-EELS technique for near-field mapping of dielectric nanocavity eigenmodes with high sensitivity. Compared with the scattering of EM illumination, there is a more direct connection between the eigenmode's nature and its EELS response, just with certain modes showing consistent peak/resonance energy discrepancies that demand further investigation. The results also give insights into how the resonances can be tailored by modifying the nanocavity size and shape.

\section{METHODS}

Initially, $100 \mathrm{~nm}$ thick a-Si dielectric nanocavities were lithographically patterned on $35 \mathrm{~nm}$ thick $\mathrm{Si}_{3} \mathrm{~N}_{4}$ membranes using the following procedure. Double-side polished $100 \mathrm{~mm}$ diameter $\mathrm{Si}$ wafers were coated with $\mathrm{Si}_{3} \mathrm{~N}_{4}$ and a-Si layers, both by low pressure chemical vapor deposition. Electron beam lithography with negative tone hydrogen silsesquioxane resist, followed by hydrogen bromide dry etching, was used to pattern and etch the Si structures. ${ }^{43}$ Further etching was used to make $\mathrm{Si}_{3} \mathrm{~N}_{4}$ membrane windows of $500 \times 1000$ $\mu \mathrm{m}^{2}$ size. Each window is surrounded by a square-shaped Si frame that can be cleaved out and inserted into a standard single tilt sample holder of the electron microscope. ${ }^{10}$ STEM-EELS maps, each having $\geq 600 \times 400$ pixel $^{2}$ dimensions, were acquired on a double aberrationcorrected FEI Titan Themis 60-300 using a Gatan GIF Quantum ERS spectrometer set to a $10 \mathrm{meV}$ per channel dispersion. The $300 \mathrm{keV}$ energy incident electron beam was monochromated in order to reduce its energy spread to $\sim 100-110 \mathrm{meV}$ full-width half-maximum, resulting in a subnm probe size. The map pixel sizes varied from 0.34 $\mathrm{nm}$ for the smallest nanocavity size ( $50 \mathrm{~nm}$ disc) up to a maximum of $2.0 \mathrm{~nm}$ for large nanocavities. The illumination setup was adjusted to give beam currents of $\sim 200-260 \mathrm{pA}$, which enables a relatively high EELS signal at the energy losses of interest while applying fast, high spatial statistics mapping with short pixel dwell times of $0.22-0.27 \mathrm{~ms}$ $\left(>1000\right.$ spectra s $\left.^{-1}\right)$.

EEL spectra and heat intensity maps are extracted from the STEMEELS mapping data cubes. The spectra are integrated from regions at different impact parameters. Mostly these are square or rectangular boxes equivalent to $60 \times 60$ pixels $^{2}$ in size. For the discs, in order to maximize signal-to-noise, the spectra are instead integrated from ringshaped regions at different radii. The displayed spectra are normalized to the zero-loss peak (ZLP) intensity and subtracted for background intensity from the $\mathrm{ZLP}$ and $\mathrm{Si}_{3} \mathrm{~N}_{4}$ membrane. The displayed heatintensity maps are integrated across $0.06 \mathrm{eV}$ wide energy windows centered on the specified energy loss value. They are normalized to the ZLP intensity, in order to recover the proper projected intensity distribution which is otherwise masked by bulk plasmon and other scattering processes. ${ }^{46}$ Noise in the presented EEL spectra and maps is reduced by smoothing using a penalized least-squares method. ${ }^{66}$

All simulations in this paper are performed with the commercial 3D EM analysis software package CST Studio Suite from Dassault Systèmes, which offers solvers including time and frequency domain methods. ${ }^{53}$ For the eigenmode calculations, we applied the finite element method based Eigenmode Solver with second-order basis functions and curved mesh elements. The simulations include the $\mathrm{Si}_{3} \mathrm{~N}_{4}$ support membrane, taking refractive indices for this and the a-Si from ellipsometry measurements, and planar dimensions for the nanocavities based on their HAADF STEM images. The eigenmode electric field distribution plots are shown when the resonances are at or near their maximum electric field values.

Further details on sample fabrication, STEM-EELS data acquisition and processing, and simulations are provided in the SI.

\section{ASSOCIATED CONTENT}

\section{SI Supporting Information}

The Supporting Information is available free of charge at https://pubs.acs.org/doi/10.1021/acsnano.1c06065.

Further details on experimental and simulation methods; a compilation of STEM-EELS data from 150, 300, and $450 \mathrm{~nm}$ discs, $450 \times 300$ and $600 \times 300 \mathrm{~nm}$ ellipse and rectangle, $1000 \times 500 \mathrm{~nm}$ rectangle, and $300 \mathrm{~nm}$ side triangle and square; results of EM simulations of eigenmodes, frequency domain dipole excitation, and far-field scattering (PDF)

Tables of optical data for the a-Si and $\mathrm{Si}_{3} \mathrm{~N}_{4}$ materials (XLSX)

\section{AUTHOR INFORMATION}

\section{Corresponding Author}

Duncan T. L. Alexander - Electron Spectrometry and Microscopy Laboratory (LSME), Institute of Physics (IPHYS), Ecole Polytechnique Fédérale de Lausanne (EPFL), 1015 Lausanne, Switzerland; 10 orcid.org/0000-00034350-8587; Phone: +41 2169 35323;

Email: duncan.alexander@epfl.ch,dtla@cantab.net

\section{Authors}

Valentin Flauraud - Microsystems Laboratory (LMIS1), Microengineering Institute (IMT), Ecole Polytechnique Fédérale de Lausanne (EPFL), 1015 Lausanne, Switzerland; (1) orcid.org/0000-0002-1393-3198

Frank Demming-Janssen - SIMUSERV GmbH, 97076 Würzburg, Germany

Complete contact information is available at: https://pubs.acs.org/10.1021/acsnano.1c06065

\section{Notes}

The authors declare no competing financial interest.

\section{ACKNOWLEDGMENTS}

EPFL's Interdisciplinary Centre for Electron Microscopy (CIME) and Center of MicroNanoTechnology (CMi) platforms are thanked for support and access to their facilities, and J. Brugger (EPFL) for support of VF during the early stages of this project. Discussions with many colleagues have contributed to progressing this work. For these, we thank $\mathrm{H}$. Lourenço-Martins (University of Göttingen, formerly LPS Orsay), G. Bernasconi (formerly EPFL), and A. Leitis and M.L. Tseng of the Hatice BIOS group at EPFL. The STEM-EELS data were originally acquired during research that received funding from the European Commission's seventh Framework Programme (FP7-ICT-2011-7) under grant agreement 288263 (NANO-VISTA).

\section{REFERENCES}

(1) Nelayah, J.; Kociak, M.; Stéphan, O.; García De Abajo, F. J.; Tencé, M.; Henrard, L.; Taverna, D.; Pastoriza-Santos, I.; Liz-Marzán, L. M.; Colliex, C. Mapping Surface Plasmons on a Single Metallic Nanoparticle. Nat. Phys. 2007, 3, 348-353. 
(2) Bosman, M.; Keast, V. J.; Watanabe, M.; Maaroof, A. I.; Cortie, M. B. Mapping Surface Plasmons at the Nanometre Scale with an Electron Beam. Nanotechnology 2007, 18, 165505.

(3) Schmidt, F.-P.; Ditlbacher, H.; Hohenester, U.; Hohenau, A.; Hofer, F.; Krenn, J. R. Dark Plasmonic Breathing Modes in Silver Nanodisks. Nano Lett. 2012, 12, 5780-5783.

(4) Schmidt, F.-P.; Ditlbacher, H.; Hohenester, U.; Hohenau, A.; Hofer, F.; Krenn, J. R. Universal Dispersion of Surface Plasmons in Flat Nanostructures. Nat. Commun. 2014, 5, 3604.

(5) Bellido, E. P.; Manjavacas, A.; Zhang, Y.; Cao, Y.; Nordlander, P.; Botton, G. A. Electron Energy-Loss Spectroscopy of Multipolar Edge and Cavity Modes in Silver Nanosquares. ACS Photonics 2016, $3,428-433$.

(6) Campos, A.; Arbouet, A.; Martin, J.; Gérard, D.; Proust, J.; Plain, J.; Kociak, M. Plasmonic Breathing and Edge Modes in Aluminum Nanotriangles. ACS Photonics 2017, 4, 1257-1263.

(7) Chu, M.-W.; Myroshnychenko, V.; Chen, C. H.; Deng, J.-P.; Mou, C.-Y.; García De Abajo, F. J. Probing Bright and Dark SurfacePlasmon Modes in Individual and Coupled Noble Metal Nanoparticles Using an Electron Beam. Nano Lett. 2009, 9, 399-404.

(8) Koh, A. L.; Fernández-Domínguez, A. I.; McComb, D. W.; Maier, S. A.; Yang, J. K. W. High-Resolution Mapping of ElectronBeam-Excited Plasmon Modes in Lithographically Defined Gold Nanostructures. Nano Lett. 2011, 11, 1323-1330.

(9) Barrow, S. J.; Rossouw, D.; Funston, A. M.; Botton, G. A.; Mulvaney, P. Mapping Bright and Dark Modes in Gold Nanoparticle Chains Using Electron Energy Loss Spectroscopy. Nano Lett. 2014, 14, 3799-3808.

(10) Flauraud, V.; Bernasconi, G. D.; Butet, J.; Alexander, D. T. L.; Martin, O. J. F.; Brugger, J. Mode Coupling in Plasmonic Heterodimers Probed with Electron Energy Loss Spectroscopy. ACS Nano 2017, 11, 3485-3495.

(11) Kirner, F.; Potapov, P.; Schultz, J.; Geppert, J.; Müller, M.; González-Rubio, G.; Sturm, S.; Lubk, A.; Sturm, E. AdditiveControlled Synthesis of Monodisperse Single Crystalline Gold Nanoparticles: Interplay of Shape and Surface Plasmon Resonance. J. Mater. Chem. C 2020, 8, 10844-10851.

(12) Schubert, I.; Sigle, W.; van Aken, P. A.; Trautmann, C.; ToimilMolares, M. E. STEM-EELS Analysis of Multipole Surface Plasmon Modes in Symmetry-Broken AuAg Nanowire Dimers. Nanoscale 2015, 7, 4935-4941.

(13) Flauraud, V.; Mastrangeli, M.; Bernasconi, G. D.; Butet, J.; Alexander, D. T. L.; Shahrabi, E.; Martin, O. J. F.; Brugger, J. Nanoscale Topographical Control of Capillary Assembly of Nanoparticles. Nat. Nanotechnol. 2017, 12, 73-80.

(14) Flauraud, V.; Bernasconi, G. D.; Butet, J.; Mastrangeli, M.; Alexander, D. T. L.; Martin, O. J. F.; Brugger, J. Mode Evolution in Strongly Coupled Plasmonic Dolmens Fabricated by Templated Assembly. ACS Photonics 2017, 4, 1661-1668.

(15) Ögüt, B.; Vogelgesang, R.; Sigle, W.; Talebi, N.; Koch, C. T.; van Aken, P. A. Hybridized Metal Slit Eigenmodes as an Illustration of Babinet's Principle. ACS Nano 2011, 5, 6701-6706.

(16) Bicket, I. C.; Bellido, E. P.; McRae, D. M.; Lagugné-Labarthet, F.; Botton, G. A. Carving Plasmon Modes in Silver Sierpiński Fractals. ACS Photonics 2019, 6, 2974-2984.

(17) Bellido, E. P.; Bernasconi, G. D.; Rossouw, D.; Butet, J.; Martin, O. J. F.; Botton, G. A. Self-Similarity of Plasmon Edge Modes on Koch Fractal Antennas. ACS Nano 2017, 11, 11240-11249.

(18) Bicket, I. C.; Bellido, E. P.; McRae, D. M.; Lagugné-Labarthet, F.; Botton, G. A. Hierarchical Plasmon Resonances in Fractal Structures. ACS Photonics 2020, 7, 1246-1254.

(19) Haberfehlner, G.; Trügler, A.; Schmidt, F. P.; Hörl, A.; Hofer, F.; Hohenester, U.; Kothleitner, G. Correlated 3D Nanoscale Mapping and Simulation of Coupled Plasmonic Nanoparticles. Nano Lett. 2015, 15, 7726-7730.

(20) Hörl, A.; Haberfehlner, G.; Trügler, A.; Schmidt, F.-P.; Hohenester, U.; Kothleitner, G. Tomographic Imaging of the Photonic Environment of Plasmonic Nanoparticles. Nat. Commun. 2017, 8, 37.
(21) Haberfehlner, G.; Schmidt, F.-P.; Schaffernak, G.; Hörl, A.; Trügler, A.; Hohenau, A.; Hofer, F.; Krenn, J. R.; Hohenester, U.; Kothleitner, G. 3D Imaging of Gap Plasmons in Vertically Coupled Nanoparticles by EELS Tomography. Nano Lett. 2017, 17, 67736777.

(22) Lourenço-Martins, H.; Das, P.; Tizei, L. H. G.; Weil, R.; Kociak, M. Self-Hybridization within Non-Hermitian Localized Plasmonic Systems. Nat. Phys. 2018, 14, 360-364.

(23) Yankovich, A. B.; Munkhbat, B.; Baranov, D. G.; Cuadra, J.; Olsén, E.; Lourenço-Martins, H.; Tizei, L. H. G.; Kociak, M.; Olsson, E.; Shegai, T. Visualizing Spatial Variations of Plasmon-Exciton Polaritons at the Nanoscale Using Electron Microscopy. Nano Lett. 2019, 19, 8171-8181.

(24) García De Abajo, F. J. Optical Excitations in Electron Microscopy. Rev. Mod. Phys. 2010, 82, 209-275.

(25) Hohenester, U.; Ditlbacher, H.; Krenn, J. R. Electron-EnergyLoss Spectra of Plasmonic Nanoparticles. Phys. Rev. Lett. 2009, 103, 106801.

(26) Hohenester, U.; Trügler, A. MNPBEM - A Matlab Toolbox for the Simulation of Plasmonic Nanoparticles. Comput. Phys. Commun. 2012, 183, 370-381.

(27) Bosman, M.; Ye, E.; Tan, S. F.; Nijhuis, C. A.; Yang, J. K. W.; Marty, R.; Mlayah, A.; Arbouet, A.; Girard, C.; Han, M.-Y. Surface Plasmon Damping Quantified with an Electron Nanoprobe. Sci. Rep. 2013, 3, 1312.

(28) Bernasconi, G. D.; Butet, J.; Flauraud, V.; Alexander, D.; Brugger, J.; Martin, O. J. F. Where Does Energy Go in Electron Energy Loss Spectroscopy of Nanostructures? ACS Photonics 2017, 4, $156-164$.

(29) Guzzinati, G.; Béché, A.; Lourenço-Martins, H.; Martin, J.; Kociak, M.; Verbeeck, J. Probing the Symmetry of the Potential of Localized Surface Plasmon Resonances with Phase-Shaped Electron Beams. Nat. Commun. 2017, 8, 14999.

(30) Lourenço-Martins, H.; Lubk, A.; Kociak, M. Bridging NanoOptics and Condensed Matter Formalisms in a Unified Description of Inelastic Scattering of Relativistic Electron Beams. SciPost Phys. 2021, 10,031 .

(31) Bharadwaj, P.; Deutsch, B.; Novotny, L. Optical Antennas. Adv. Opt. Photonics 2009, 1, 438-483.

(32) Biagioni, P.; Huang, J.-S.; Hecht, B. Nanoantennas for Visible and Infrared Radiation. Rep. Prog. Phys. 2012, 75, 024402.

(33) Decker, M.; Staude, I. Resonant Dielectric Nanostructures: A Low-Loss Platform for Functional Nanophotonics. J. Opt. 2016, 18, 103001.

(34) van de Groep, J.; Polman, A. Designing Dielectric Resonators on Substrates: Combining Magnetic and Electric Resonances. Opt. Express 2013, 21, 26285-26302.

(35) Staude, I.; Miroshnichenko, A. E.; Decker, M.; Fofang, N. T.; Liu, S.; Gonzales, E.; Dominguez, J.; Luk, T. S.; Neshev, D. N.; Brener, I.; Kivshar, Y. Tailoring Directional Scattering through Magnetic and Electric Resonances in Subwavelength Silicon Nanodisks. ACS Nano 2013, 7, 7824-7832.

(36) van de Haar, M. A.; van de Groep, J.; Brenny, B. J. M.; Polman, A. Controlling Magnetic and Electric Dipole Modes in Hollow Silicon Nanocylinders. Opt. Express 2016, 24, 2047-2064.

(37) Kerker, M.; Wang, D.-S.; Giles, C. L. Electromagnetic Scattering by Magnetic Spheres. J. Opt. Soc. Am. 1983, 73, 765-767.

(38) Person, S.; Jain, M.; Lapin, Z.; Sáenz, J. J.; Wicks, G.; Novotny, L. Demonstration of Zero Optical Backscattering from Single Nanoparticles. Nano Lett. 2013, 13, 1806-1809.

(39) Fu, Y. H.; Kuznetsov, A. I.; Miroshnichenko, A. E.; Yu, Y. F.; Luk'yanchuk, B. Directional Visible Light Scattering by Silicon Nanoparticles. Nat. Commun. 2013, 4, 1527.

(40) Miroshnichenko, A. E.; Evlyukhin, A. B.; Yu, Y. F.; Bakker, R. M.; Chipouline, A.; Kuznetsov, A. I.; Luk'yanchuk, B.; Chichkov, B. N.; Kivshar, Y. S. Nonradiating Anapole Modes in Dielectric Nanoparticles. Nat. Commun. 2015, 6, 8069.

(41) Powell, D. A. Interference between the Modes of an AllDielectric Meta-Atom. Phys. Rev. Appl. 2017, 7, 034006. 
(42) Baryshnikova, K. V.; Smirnova, D. A.; Luk'yanchuk, B. S.; Kivshar, Y. S. Optical Anapoles: Concepts and Applications. Adv. Opt. Mater. 2019, 7, 1801350.

(43) Flauraud, V.; Reyes, M.; Paniagua-Domínguez, R.; Kuznetsov, A. I.; Brugger, J. Silicon Nanostructures for Bright Field Full Color Prints. ACS Photonics 2017, 4, 1913-1919.

(44) García de Abajo, F. J.; Pattantyus-Abraham, A. G.; Zabala, N.; Rivacoba, A.; Wolf, M. O.; Echenique, P. M. Cherenkov Effect as a Probe of Photonic Nanostructures. Phys. Rev. Lett. 2003, 91, 143902.

(45) Cha, J. J.; Yu, Z.; Smith, E.; Couillard, M.; Fan, S.; Muller, D. A. Mapping Local Optical Densities of States in Silicon Photonic Structures with Nanoscale Electron Spectroscopy. Phys. Rev. B: Condens. Matter Mater. Phys. 2010, 81, 113102.

(46) Le Thomas, N.; Alexander, D. T. L.; Cantoni, M.; Sigle, W.; Houdré, R.; Hébert, C. Imaging of High-Q Cavity Optical Modes by Electron Energy-Loss Microscopy. Phys. Rev. B: Condens. Matter Mater. Phys. 2013, 87, 155314.

(47) Habteyes, T. G.; Staude, I.; Chong, K. E.; Dominguez, J.; Decker, M.; Miroshnichenko, A.; Kivshar, Y.; Brener, I. Near-Field Mapping of Optical Modes on All-Dielectric Silicon Nanodisks. ACS Photonics 2014, 1, 794-798.

(48) Coenen, T.; van de Groep, J.; Polman, A. Resonant Modes of Single Silicon Nanocavities Excited by Electron Irradiation. ACS Nano 2013, 7, 1689-1698.

(49) van de Groep, J.; Coenen, T.; Mann, S. A.; Polman, A. Direct Imaging of Hybridized Eigenmodes in Coupled Silicon Nanoparticles. Optica 2016, 3, 93-99.

(50) Matsukata, T.; Matthaiakakis, N.; Yano, T.; Hada, M.; Tanaka, T.; Yamamoto, N.; Sannomiya, T. Selection and Visualization of Degenerate Magnetic and Electric Multipoles up to Radial Higher Orders by Cathodoluminescence. ACS Photonics 2019, 6, 2320-2326.

(51) Liu, Q.; Quillin, S. C.; Masiello, D. J.; Crozier, P. A. Nanoscale Probing of Resonant Photonic Modes in Dielectric Nanoparticles with Focused Electron Beams. Phys. Rev. B: Condens. Matter Mater. Phys. 2019, 99, 165102.

(52) Koh, A. L.; Bao, K.; Khan, I.; Smith, W. E.; Kothleitner, G.; Nordlander, P.; Maier, S. A.; McComb, D. W. Electron Energy-Loss Spectroscopy (EELS) of Surface Plasmons in Single Silver Nanoparticles and Dimers: Influence of Beam Damage and Mapping of Dark Modes. ACS Nano 2009, 3, 3015-3022.

(53) CST Studio Suite, Release 2020.07; Dassault Systèmes: VélizyVillacoublay, France, 2020.

(54) Nicoletti, O.; de la Peña, F.; Leary, R. K.; Holland, D. J.; Ducati, C.; Midgley, P. A. Three-Dimensional Imaging of Localized Surface Plasmon Resonances of Metal Nanoparticles. Nature 2013, 502, 80-84.

(55) Cao, Y.; Manjavacas, A.; Large, N.; Nordlander, P. Electron Energy-Loss Spectroscopy Calculation in Finite-Difference TimeDomain Package. ACS Photonics 2015, 2, 369-375.

(56) Losquin, A.; Kociak, M. Link between Cathodoluminescence and Electron Energy Loss Spectroscopy and the Radiative and Full Electromagnetic Local Density of States. ACS Photonics 2015, 2, $1619-1627$.

(57) Kajfez, D.; Glisson, A. W.; James, J. Computed Modal Field Distributions for Isolated Dielectric Resonators. IEEE Trans. Microwave Theory Tech. 1984, 32, 1609-1616.

(58) Lepetit, T.; Kanté, B. Controlling Multipolar Radiation with Symmetries for Electromagnetic Bound States in the Continuum. Phys. Rev. B: Condens. Matter Mater. Phys. 2014, 90, 241103.

(59) Alber, I.; Sigle, W.; Demming-Janssen, F.; Neumann, R.; Trautmann, C.; van Aken, P. A.; Toimil-Molares, M. E. Multipole Surface Plasmon Resonances in Conductively Coupled Metal Nanowire Dimers. ACS Nano 2012, 6, 9711-9717.

(60) Yang, Y.; Miroshnichenko, A. E.; Kostinski, S. V.; Odit, M.; Kapitanova, P.; Qiu, M.; Kivshar, Y. S. Multimode Directionality in All-Dielectric Metasurfaces. Phys. Rev. B: Condens. Matter Mater. Phys. 2017, 95, 165426.

(61) Boudarham, G.; Kociak, M. Modal Decompositions of the Local Electromagnetic Density of States and Spatially Resolved
Electron Energy Loss Probability in Terms of Geometric Modes. Phys. Rev. B: Condens. Matter Mater. Phys. 2012, 85, 245447.

(62) Kuznetsov, A. I.; Miroshnichenko, A. E.; Fu, Y. H.; Zhang, J.; Luk'yanchuk, B. Magnetic Light. Sci. Rep. 2012, 2, 492.

(63) Mühlig, S.; Menzel, C.; Rockstuhl, C.; Lederer, F. Multipole Analysis of Meta-Atoms. Metamaterials 2011, 5, 64-73.

(64) Schmidt, F.-P.; Losquin, A.; Hofer, F.; Hohenau, A.; Krenn, J. R.; Kociak, M. How Dark Are Radial Breathing Modes in Plasmonic Nanodisks? ACS Photonics 2018, 5, 861-866.

(65) Kordahl, D.; Alexander, D. T. L.; Dwyer, C. Waveguide Modes Spatially Resolved by Low-Loss STEM-EELS. Phys. Rev. B: Condens. Matter Mater. Phys. 2021, 103, 134109.

(66) Garcia, D. Robust Smoothing of Gridded Data in One and Higher Dimensions with Missing Values. Comput. Stat. Data Anal. 2010, 54, 1167-1178. 